Check for updates

Cite this: RSC Adv., 2021, 11, 7078

Received 7th December 2020 Accepted 2nd February 2021

DOI: 10.1039/d0ra10317a

rsc.li/rsc-advances

\section{The inhibition performance of a novel benzenesulfonamide-based benzoxazine compound in the corrosion of X60 carbon steel in an acidizing environment}

\author{
Khalid A. Alamry, (D) *a Mahmoud A. Hussein, (D)*ab Abdulrahman Musa, ${ }^{a}$ \\ Kabiru Haruna ${ }^{\text {cd }}$ and Tawfik A. Saleh ${ }^{c}$
}

\begin{abstract}
A benzenesulfonamide-based benzoxazine compound (BSB) was synthesized from sulfamethizole, salicylaldehyde, and paraformaldehyde in a series of reaction steps. The novel phenolic compound was structurally identified by spectroscopic techniques such as nuclear magnetic resonance $\left({ }^{1} \mathrm{H} \&{ }^{13} \mathrm{C}-\mathrm{NMR}\right)$ and Fourier transform infrared spectroscopy. This novel benzoxazine compound possesses some highly electronegative atoms that are responsible for its corrosion inhibition behavior. The corrosion inhibition performance of $\mathrm{BSB}$ against $\mathrm{X} 60$ steel corrosion in $15 \% \mathrm{HCl}$ at 25,40 , and $60{ }^{\circ} \mathrm{C}$, which mimics an oilwell acidizing environment, was investigated by weight loss (WL) measurements and electrochemical techniques. The surface chemical compositions of the inhibitor and substrate (before and after corrosion) were examined using energy dispersive X-ray (EDX), and Fourier transform infrared spectroscopy (FTIR) techniques, respectively. Structural analysis was performed on the corroded steel samples, by scanning electron microscopy (SEM), and atomic force microscopy (AFM) measurements. The WL study of BSB at room temperature revealed over $88 \%$ inhibition efficiency for its $600 \mathrm{ppm}$ concentration at room temperature and the efficiency slightly increased to $91 \%$ at $60{ }^{\circ} \mathrm{C}$. Furthermore, the study suggested adsorption of BSB at a concentration of $600 \mathrm{ppm}$ could involve the chemisorption adsorption mechanism. However, the PDP results at all the studied concentrations revealed BSB to act as a mixed-type inhibitor with a cathodic predominance. The EDS and FTIR analyses confirmed the BSB adsorption to the steel surface to have occurred via interactions between the BSB heteroatoms and the carbon steel surface. Hence, BSB adsorption follows the Langmuir adsorption isotherm.
\end{abstract}

\section{Introduction}

Carbon steel is an essential, industrial raw material manufactured as an alloy of iron, carbon, and some trace amounts of heavy metals. ${ }^{1}$ Carbon steel has excellent welding, mechanical and annealing characteristics, and is also available at a low cost. $^{2}$ Carbon steel exhibits excellent qualities that satisfy its application in the construction industry, and oil and gas sectors. ${ }^{3-5}$ Mineral acids such as hydrofluoric acid (HF), nitric acid $\left(\mathrm{HNO}_{3}\right)$, hydrochloric acid $(\mathrm{HCl})$, and organic acids; formic acid $(\mathrm{HCOOH})$, acetic acid $\left(\mathrm{CH}_{3} \mathrm{COOH}\right)$, chloroacetic acid

${ }^{a}$ Chemistry Department, Faculty of Science, King Abdulaziz University, P.O. Box 80203, Jeddah 21589, Saudi Arabia. E-mail: kaalamri@kau.edu.sa; mahussein74@yahoo. com;maabdo@kau.edu.sa

${ }^{b}$ Polymer Chemistry Lab., Chemistry Department, Faculty of Science, Assiut University, Assiut, 71516, Egypt

${ }^{c}$ Department of Chemistry, King Fahd University of Petroleum and Minerals, Dhahran 31261, Saudi Arabia

${ }^{d}$ Centre for Engineering Research, Research Institute, King Fahd University of Petroleum and Minerals, Dhahran 31261, Saudi Arabia
$\left(\mathrm{ClCH}_{2} \mathrm{COOH}\right)$, sulfonic acid $\left(\mathrm{RSO}_{2} \mathrm{OH}\right)$, and sulfamic acid $\left(\mathrm{H}_{2} \mathrm{NSO}_{2} \mathrm{OH}\right)$, are used to dissolve rubble, remove mud and to create channels through rocks to access the crude oil. ${ }^{6}$ The carbon steel employed for oil explorations in wells is susceptible to corrosion, due to the harsh working conditions of the oil wells, with acid solutions used as solvents to dissolve the rocks at the drilling sites. Today, this practice is adopted worldwide to enhance large oil and gas production, and the method of using $(5-28 \%) \mathrm{HCl}$ is reported as the most prominent. $^{7}$

Corrosion is an electrochemical and metallurgical process that causes tremendous damages to construction materials, constituting industrial problems such as short-circuit of the electrical systems, leakages of tanks and pipelines. The annual cost of mitigating corrosion in the industrialized world's according to ${ }^{8}$ is about $£ 1.3$ billion, and this estimated amount represents nearly $3-4 \%$ of their GDP per annum., ${ }^{\mathbf{9} 10}$ Corrosion has been controlled by some methods that include coating the surfaces of the corrosive materials with metals and organic polymers, ${ }^{11-16}$ the use of inorganic and organic compounds as corrosion inhibitors, and cathodic protection. ${ }^{17}$ In the oil wells, 
the use of corrosion inhibitors has proven to be easily practicable, efficient, and financially more economical. ${ }^{6}$ The inhibitors are adsorbed to form protective layers over the surface of the substrates, to prevent it from the ions of the corrosion environment. ${ }^{18}$ Some inorganic inhibitors such as salts of nitrate $\left(\mathrm{NO}_{2}{ }^{-}\right)$, molybdate, $\left(\mathrm{MoO}_{3}{ }^{-}\right)$, chromate $\left(\mathrm{CrO}_{4}{ }^{2-}\right)$, phosphate $\left(\mathrm{H}_{2} \mathrm{PO}_{3}{ }^{-}\right)$, and silicate, in the presence of arsenic, zinc, nickel, and copper have been used to mitigate corrosion at high temperature with efficient performance and are relatively inexpensive compared to the organic inhibitors. However, inorganic inhibitors are non-biodegradable with tougher tendency to combine with metal substrates. They also have more possibilities of losing grip at high acid concentrations beyond $17 \%$ compared to the organic inhibitors. ${ }^{9}$ Environmental concerns are associated with such inhibitors obtained from arsenic salts that tend to release toxic arsine gas. ${ }^{19}$ The organic inhibitors with hetero-atoms, show excellent affinity to the surface of the metallic substrates, due to the strong interaction between the pi-electrons of these atoms, and the empty d-orbitals of the transition metal (Fe atoms). ${ }^{3,20}$ This interaction causes the formation of a protected layer over the metal surface, that prevents it from direct contact with the corrosive environment. ${ }^{21}$ The heterocyclic compounds, whose structures contain double bonds, and also bearing hetero-atoms such as, nitrogen $(\mathrm{N})$, sulphur $(\mathrm{S})$, phosphorus $(\mathrm{P})$, and oxygen $(\mathrm{O})$, are considered the most effective organic inhibitors for the corrosion steel materials. $^{22}$ Compounds containing functional absorption centers such as; $-\mathrm{SO}_{2}-\mathrm{NH}$ - groups, $-\mathrm{NH}_{2}$ group, aromatic rings, are referred to as sulfa drugs and have been reported to exhibit excellent corrosion inhibition performance because of the presence of these non-carbon atoms in them. ${ }^{20}$

Benzoxazines are heterocyclic organic compounds formed by reactions of phenols, amines, and paraformaldehyde. ${ }^{23}$ Cope and Holy in the 1940s were the first to synthesize this class of compound. ${ }^{24}$ Benzoxazines, majorly used as monomers for the synthesis of polybenzoxazines, ${ }^{23}$ and are reported to show excellent corrosion inhibition behavior when tested for this application. The corrosion inhibition properties of benzoxazines are related to the existence of an oxazine heterocycle connected to a benzene ring in their primary chemical structure, ${ }^{25}$ and the oxazine ring contains heteroatoms (nitrogen $(\mathrm{N})$ and oxygen $(\mathrm{O})$ ) that bear electron pairs. This investigation is on the synthesis of a benzenesulfonamide functionalized benzoxazine (BSB), and the effect of the functional group of the compound on its corrosion inhibition efficacy. The BSB structure has additional electron-rich; sulphur, nitrogens, and oxygen atoms. The compound obtained by reacting salicylaldehyde, sulfamethizole, and paraformaldehyde, was analyzed by some techniques. FTIR and NMR spectra of the product were examined to confirm its chemical makeup. Scanning electron microscopy (SEM), was used to characterize the surface morphology of the compound. The quantification of the elemental distribution on the surface of BSB by energy dispersive X-rays (EDX) was also investigated. Then, the inhibition study of BSB against a substrate of steel (X60) using a solution of $15 \% \mathrm{HCl}$ simulating an acidizing environment was examined, by weight loss analysis and electrochemical measurements. The surface characterization techniques such as SEM/EDS, AFM, and FTIR, were used to analyze the surfaces of the substrates tested in the BSB and blank solutions.

\section{Experimental}

\subsection{Materials}

Sulfamethizole (Sigma-Aldrich, 98\%), salicylaldehyde (SigmaAldrich, $\geq 98 \%$ ), paraformaldehyde (Sigma-Aldrich, 95\%), sodium borohydride (Sigma-Aldrich, 99\%), carbon steel (X60), ethanol (Sigma-Aldrich, $\geq 99.5 \%$ ), hydrochloric acid (SigmaAldrich, 37\%), methanol (Sigma-Aldrich, 98\%), and hexamethylene tetraamine (Sigma-Aldrich, $\geq 99 \%$ ). All the chemicals were used as supplied without additional purifications.

\subsection{Inhibitor synthesis}

The inhibitor employed for this study was synthesized in two reaction steps, as depicted in Fig. 1. Firstly, a phenol-derivative BSP, was prepared via the reaction of an ortho-

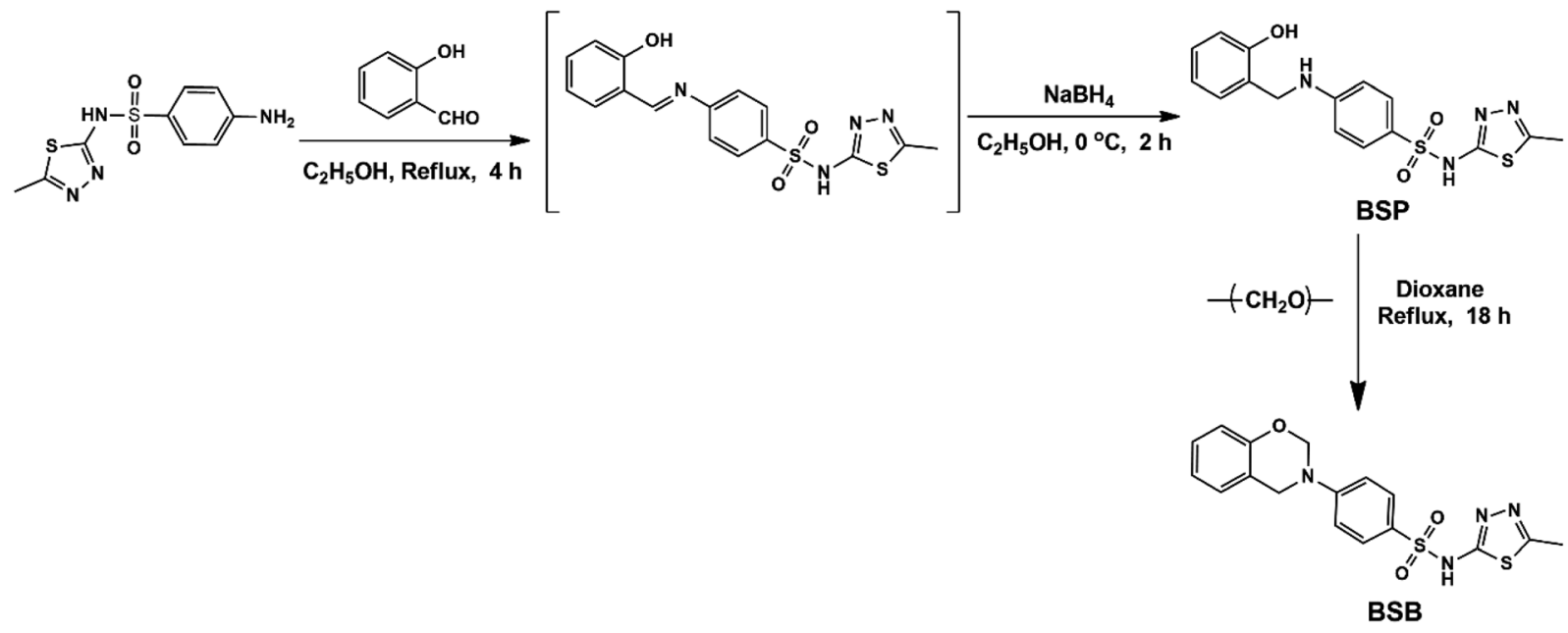

Fig. 1 Schematic showing the synthesis of benzenesulfonamide-based benzoxazine (BSB). 
hydroxybenzaldehyde and a primary amine. This reaction was performed according to the methodology reported by Fei Shan and his colleagues. ${ }^{21}$ Then, at the final step, the targeted benzenesulfonamide-based benzoxazine (BSB) was obtained by the reaction of 4-((2-hydroxybenzyl)amino)- $N$-(5-methyl-1,3,4thiadiazol-2-yl)benzenesulfonamide precursor abbreviated as (BSP), with formaldehyde molecules.

2.2.1. Synthesis of BSP. A $250 \mathrm{~cm}^{-3}$ round-bottom flask, containing a magnet stirrer, was filled with $120 \mathrm{~cm}^{-3}$ of ethanol. Then, sulfamethizole (4.88 g, 0.04 moles), and salicylaldehyde (10.80 g, 0.04 moles), were dissolved in the solvent and refluxed for 4 hours. Sodium borohydride $(2.22 \mathrm{~g}, 0.06$ mole) was added to this reaction flask and ice-cooled at $0{ }^{\circ} \mathrm{C}$. The mixture was gently stirred for 2 hours at this temperature before discharging into $450 \mathrm{~cm}^{-3}$ water. The neutralization of the aqueous solution was achieved with $10 \% \mathrm{HCl}$ to give a white-powered precipitate of BSP. The collected product was severally washed with water and dried overnight at $50{ }^{\circ} \mathrm{C}$ in a vacuum oven before used for the next reaction step. Yield $90 \%, \mathrm{mp}=203{ }^{\circ} \mathrm{C}$. FTIR spectroscopy (KBr 4000-400 cm $\left.{ }^{-1}\right)$ : $3366(\mathrm{O}-\mathrm{H}), 3095-3140$ (thiazole ring), 1593 (aromatic $\mathrm{C}=\mathrm{C}$ ),1397 (asymmetric $\mathrm{S}=\mathrm{O}$ ), 1127 (symmetric $\mathrm{S}=\mathrm{O}) .{ }^{1} \mathrm{H}-\mathrm{NMR}[400 \mathrm{MHz}, \mathrm{DMSO}, \delta(\mathrm{ppm})]: 13.62(\mathrm{~s}$, $\left.1 \mathrm{H}, \mathrm{SO}_{2}-\mathrm{NH}-\right), 9.72(\mathrm{~s}, 1 \mathrm{H},-\mathrm{OH}), 7.45\left(\mathrm{t}, 1 \mathrm{H}, \mathrm{Ar}-\mathrm{CH}_{2}-\mathrm{NH}\right), 6.60-$ $7.45(\mathrm{~m}, 8 \mathrm{H}, \mathrm{Ar}-\mathrm{H}), 4.23\left(\mathrm{~d}, 2 \mathrm{H}, \mathrm{Ar}-\mathrm{CH}_{2}-\mathrm{N}\right), 2.51$ (s, 3H, -C$\left.\mathrm{CH}_{3}\right) .{ }^{13} \mathrm{C}-\mathrm{NMR}$ [400 MHz, DMSO, $\delta$ (ppm)]: 167.61, 155.71, $154.67,152.67,128.82,128.48,128.21,125.36,119.52,115.62$, $111.67,57.78,16.73$.

2.2.2. Synthesis of 4 -(2h-benzo[e][1,3]oxazin-3(4H)-yl)-N-(5methyl-1,3,4-thiadiazol-2-yl)ben-zenesulfonamide BSB. The BSP (3.55 g, 0.00944 moles) and paraformaldehyde (0.283 g, 0.00944 moles) were dissolved in a round-bottom flask containing dioxane $\left(80 \mathrm{~cm}^{-3}\right)$ and refluxed for $18 \mathrm{~h}$. Then, the dioxane was removed under vacuum using rotary evaporator. The crude product dissolved in methylene chloride was washed with 1 molar solution of sodium hydroxide solution and rinsed severally with deionized water. Anhydrous magnesium sulphate was used to dry the organic phase and the solution left to dry at room temperature to give a dirty white solid product of BSB. The product was further purified by recrystallization in ethanol solution, yield $68 \% . \mathrm{Mp}=185^{\circ} \mathrm{C}$. FTIR spectroscopy $(\mathrm{KBr} 4000-$ $400 \mathrm{~cm}^{-1}$ ): $3342(\mathrm{~N}-\mathrm{H}), 1591$ (aromatic $\left.\mathrm{C}=\mathrm{C}\right), 1501$ (trisubstituted aromatic ring), 1371 (asymmetric $\mathrm{S}=\mathrm{O}$ ), 1272 (asymmetric C-O-C), 1127 (symmetric $\mathrm{S}=\mathrm{O}$ ), 947 (oxazine ring). ${ }^{1} \mathrm{H}$ NMR [400 MHz, DMSO, $\delta$ (ppm)]: 13.59 (s, 1H, $\left.\mathrm{SO}_{2}-\mathrm{NH}-\right)$, 6.59-7.54 (m, 8H, Ar-H), 5.89 (d, 2H, O- $\left.\mathrm{CH}_{2}-\mathrm{N}\right), 4.75$ (d, 2H, Ar$\left.\mathrm{CH}_{2}-\mathrm{N}\right), 2.51\left(\mathrm{~s}, 3 \mathrm{H},-\mathrm{C}-\mathrm{CH}_{3}\right) .{ }^{13} \mathrm{C}-\mathrm{NMR}$ [400 MHz, DMSO, $\delta(\mathrm{ppm})]:$ 165.34, 155.48, 154.64, 149.29, 130.23, 128.48, 128.14, 127.56, 123.36, 119.50, 115.57, 113.16, 64.56, 50.58, 16.77.

\subsection{Preparation of the BSB inhibitor solutions and carbon steel coupons}

Firstly, an analytical grade $\mathrm{HCl}(37 \%)$ stock solution was used to prepare an aqueous solution of $\mathrm{HCl}(15 \%)$ by the dilution method. Then, BSB solutions $(100,200,300,400,500$, and 600 $\mathrm{ppm}$ ) were individually prepared in the dilute acid solution and used for this examination.
The chemical compositions of the carbon steel specimen used for this investigation are $96.2 \% \mathrm{Fe}, 0.121 \% \mathrm{Cr}, 0.125 \% \mathrm{C}$, $0.52 \% \mathrm{Si}, 1.830 \% \mathrm{Mn}, 0.296 \% \mathrm{Cu}, 0.091 \% \mathrm{Ni}, 0.079 \% \mathrm{Mo}$, $0.043 \% \mathrm{Al}, 0.053 \% \mathrm{Nb}$ and $0.078 \% \mathrm{~V}$. The dimension $(3 \mathrm{~cm} \times$ $3 \mathrm{~cm} \times 1 \mathrm{~cm}$ ) of the specimen was mechanically fabricated into coupons for weight loss analysis. While the coupon, used for electrochemical assessments, was constructed by cold mounting $1 \mathrm{~cm} \times 1 \mathrm{~cm} \times 1 \mathrm{~cm}$ carbon steel specimen in a mixture containing epoxy and its hardener exposing only the $1 \mathrm{~cm} \times 1 \mathrm{~cm}$ square surface to give a total exposed surface area of $1 \mathrm{~cm}^{2}$. However, the coupons before usage were wet-polished with silicon carbide paper: $120,240,320,400,600$, and 800 grits. Finally, distilled water and acetone were used to rinse the coupons, and then dried using a specimen dryer.

\subsection{BSB and steel surface characterization}

The structure of the BSB inhibitor was examined using FTIRspectrophotometer (Shimadzu-8400S), within a scanning range of $400-4000 \mathrm{~cm}^{-1}$ at a resolution of $\pm 4 \mathrm{~cm}^{-1}$. FTIR spectra of the BSB film formed on the carbon steel substrates were also collected, after $24 \mathrm{~h}$ immersion of the steel in the acidic solution with, and without the BSB inhibitor. The NMR spectra $\left({ }^{1} \mathrm{H} \&{ }^{13} \mathrm{C}\right)$ the BSB sample were analyzed using a Bruker NMR spectrophotometer (400 MHz). The surfaces of the steel substrates in the presence and absence of the inhibitor were examined using the SEM instrument (JSM-6610 LV model) at an operating voltage and irradiation current of $10 \mathrm{kV}$ and $10 \mathrm{~mA}$, respectively, and the Fiber-Lite MI-150 high-intensity illuminator from Dolan-Jenner Industries AFM instrument.

\subsection{Weight loss evaluation}

Weight loss analysis was performed according to the standard procedure: American Society for Testing and Materials (ASTM) G1-03 method. ${ }^{26}$ Here, weighed samples of the steel substrates were immersed (in duplicates) into $100 \mathrm{~cm}^{3}$ of test solution contained in glass bottles $\left(200 \mathrm{~cm}^{3}\right.$ capacity). Then, the blank and different concentrations of the BSB inhibitor were added separately to the bottles and maintained at room temperature $(25 \pm 1)$, for $24 \mathrm{~h}$. Thereafter, the samples were removed from the bottles, and dipped in Clark's solution (a $3.5 \mathrm{~g}$ hexamethylene tetraamine in $1000 \mathrm{~cm}^{3} 18.5 \% \mathrm{HCl}$ solution), for 2 minutes, before totally washing them with water \& detergent. Finally, weights of the dried specimens were measured, after rinsing them with distilled water and acetone, respectively. The difference in the specimens' weights, before and after immersion in the test solution, was taken as the weight loss, and the corrosion rate (CR) calculated using eqn (1).

$$
\text { Corrosionrate }(\mathrm{mm} \text { per year })=\frac{W \times 8.76 \times 10^{4}}{A \times T \times D}
$$

where, $W, A, T$ and $D$, are the weight loss in gram, the total surface area exposed in $\mathrm{cm}^{2}$, the exposure time in hours, and the density of the steel substrate in $\mathrm{g} \mathrm{cm}^{-2}$, respectively.

The inhibition efficacy expressed as (\%IE) was estimated using eqn (2) 


$$
\% \mathrm{IE}_{\mathrm{Wt} \text { loss }}=\frac{\mathrm{CR}_{\mathrm{o}}-\mathrm{CR}_{\mathrm{I}}}{\mathrm{CR}_{\mathrm{o}}} \times 100
$$

where: $\mathrm{CR}_{\mathrm{O}}$ indicates corrosion rates for the blank, and $\mathrm{CR}_{\mathrm{I}}$ represents the corrosion rate for BSB inhibited solutions, respectively.

\subsection{Electrochemical measurement techniques}

The electrochemical evaluation of the specimen performed using a three electrodes system is reported here. The electrodes are a graphite counter electrode, a silver/silver chloride (Ag/ $\mathrm{AgCl})$ reference electrode, and a fabricated carbon steel specimen as working electrodes. The electrode system connected to an Autolab instrument (PGSTAT100N), according to ASTM standard procedure, ${ }^{26}$ was used. The corrosion characteristics of the steel substrates, in the acidizing environment $(15 \% \mathrm{HCl})$, at 25,40 , and $60^{\circ} \mathrm{C}$, was studied using the three electrochemical techniques: electrochemical impedance spectroscopy (EIS), potentiodynamic polarization (PDP), and linear polarization resistance (LPR), respectively. Before each electrochemical evaluation, the carbon steel electrode was dipped in the analysis solution for 1 hour, until a steady state is attained at open circuit potential (OCP). The EIS measurement was performed at a frequency of $100 \mathrm{kHz}$ to $10 \mathrm{mHz}$, and an amplitude of $10 \mathrm{mV}$. The LPR measurement was achieved using a voltage range of $\pm 0.01 \mathrm{~V}$ from OCP, and a $0.125 \mathrm{mV} \mathrm{s}^{-1}$ scan rate for the OCP. A voltage ranging from -0.25 to $+0.25 \mathrm{~V}$, at a scan rate of $0.25 \mathrm{mV}$ $\mathrm{s}^{-1}$, from OCP was applied for the PDP. The curve fittings and data analyses were achieved using the Nova software (2.1.4 version).

\section{Results and discussion}

\subsection{The chemistry and spectral analysis of BSB}

The benzenesulfonamide based benzoxazine (BSB) is a class of heterocyclic compounds consisting of a benzene ring attached to an oxazine heterocycle with three heteroatoms (N, O, and S), distributed on the structure of the inhibitor. The synthesis and chemical structure of the BSB are presented in Fig. 1. Firstly, a precursor material (BSP) employed for the synthesis of BSB

(a)

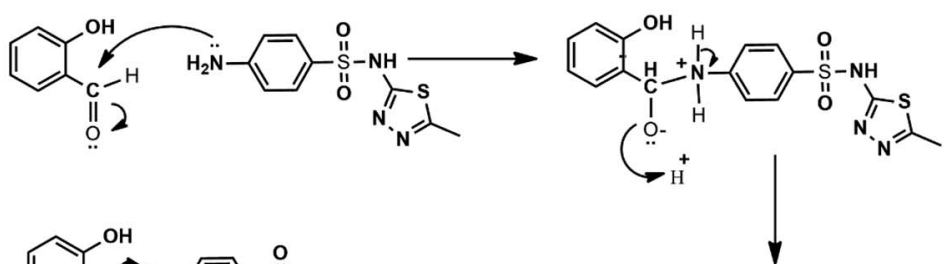

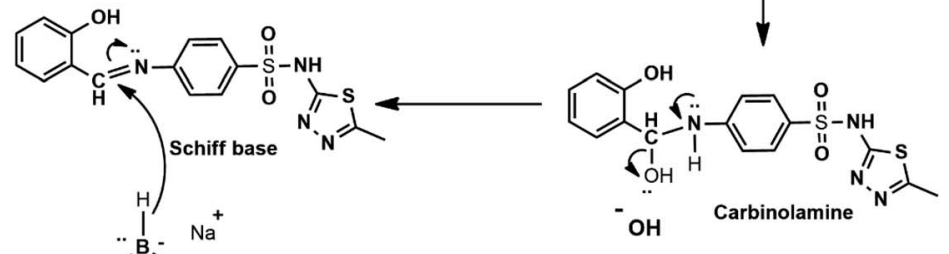
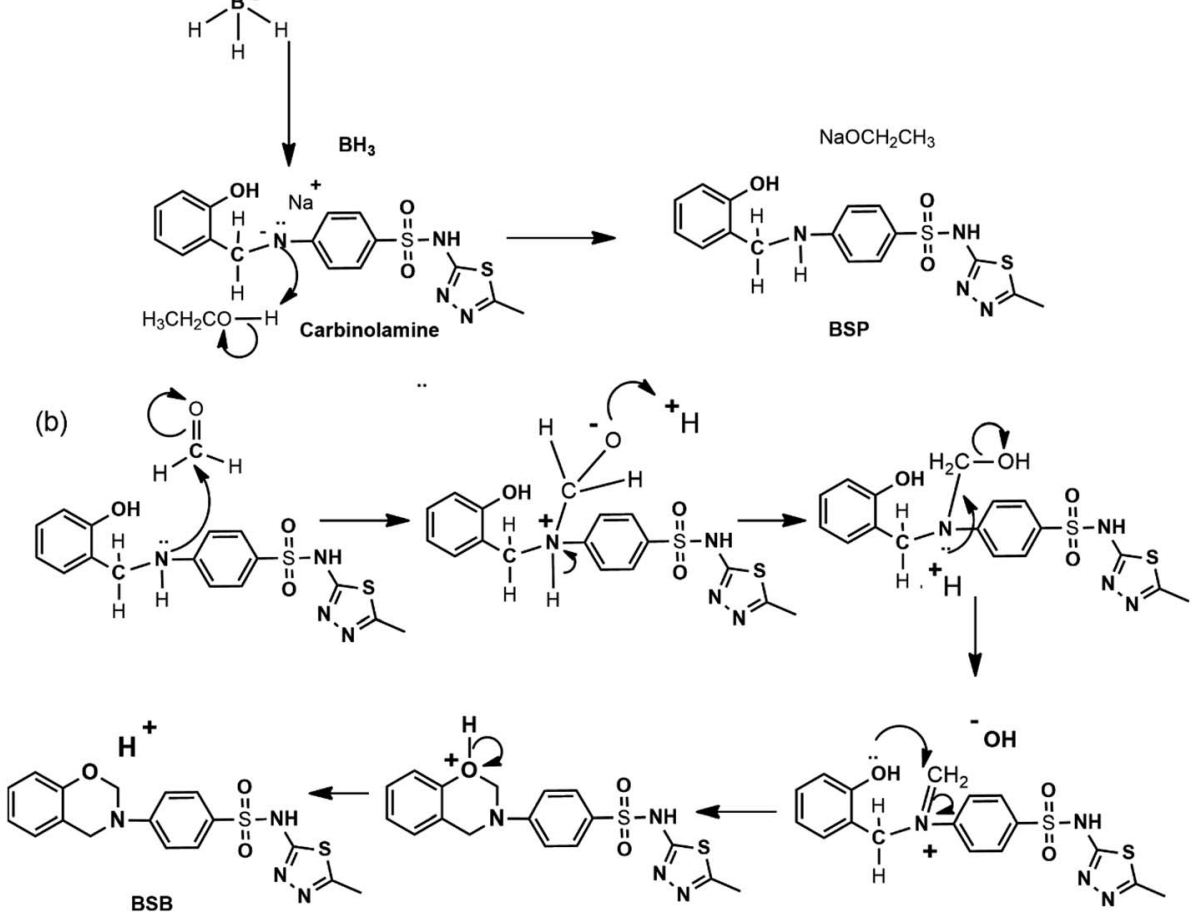

Fig. 2 Proposed mechanisms for the synthesis of BSP (a), and BSB (b). 


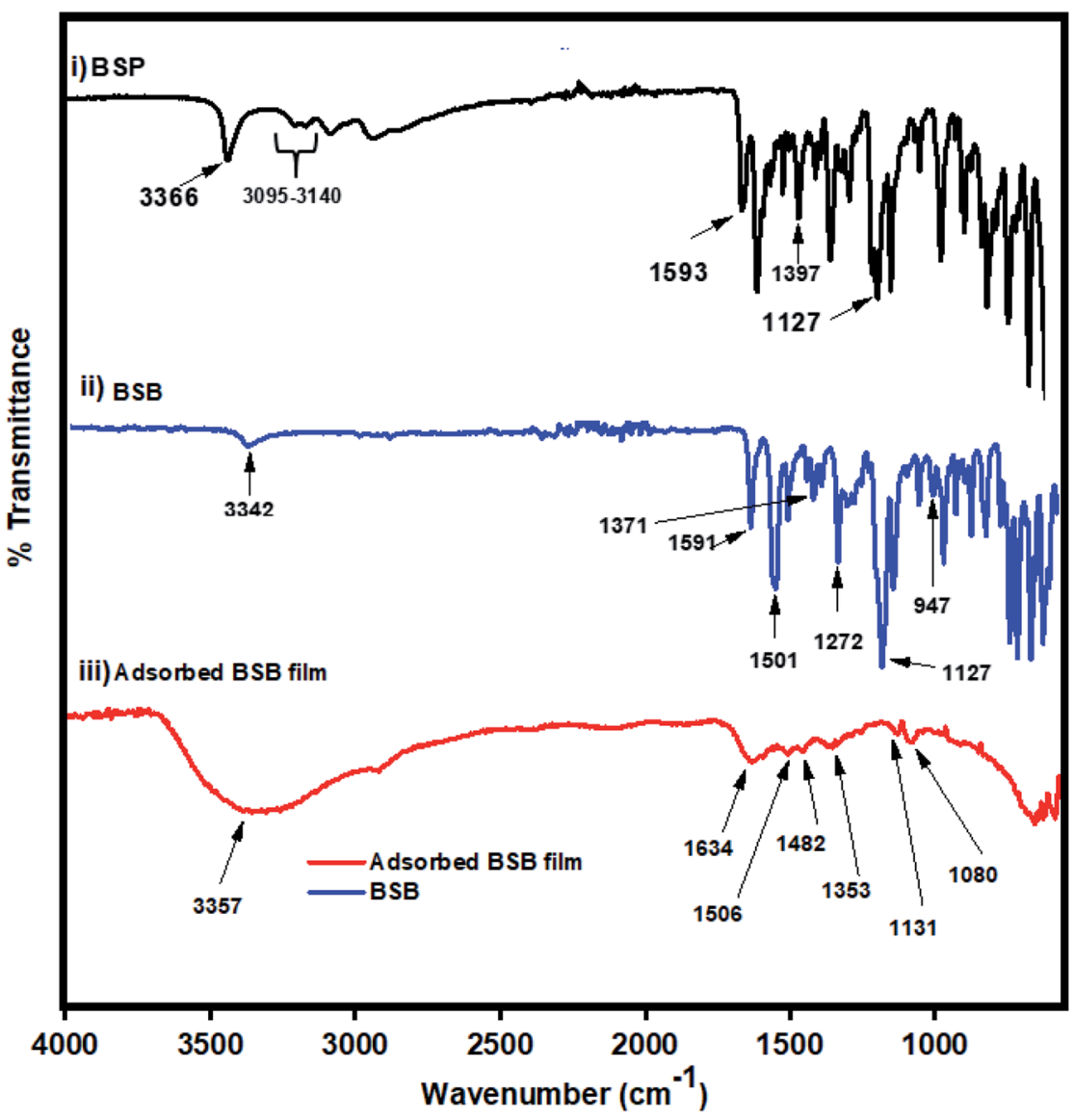

Fig. 3 FTIR spectra of BSP, BSB and carbon steel surface adsorbed BSB film.

was prepared from the chemical reduction of a Schiff-base intermediate, formed from refluxing equimolar amounts of salicylaldehyde and sulfamethizole in ethanol for 6 hours according to the methodology of Fei Shan, ${ }^{27}$ and the proposed reaction mechanism is presented in Fig. 2a. The precursor (BSP) was collected as a white powdered precipitate, dried, and characterized accordingly using FTIR, and NMR spectral analysis, respectively. From the FTIR spectra of BSP Fig. 3(i), the bands of the phenolic hydroxyl group, and the thiazole ring appeared around 3366 and $3120 \mathrm{~cm}^{-1} \cdot{ }^{28,29}$ The $\mathrm{C}=\mathrm{C}$ stretch of the aromatic rings showed at $1593 \mathrm{~cm}^{-1}$, while the vibration frequencies of the $\mathrm{S}=\mathrm{O}$ (asymmetric and symmetric), are seen at 1397 , and $1127 \mathrm{~cm}^{-1}$, respectively. ${ }^{30}$ The protons contained in BSP and the carbon centers of the compound are also examined using the NMR analysis. According to the result of the proton NMR of BSP (Fig. 5), the $\mathrm{N}-\mathrm{H}$ proton attached to the

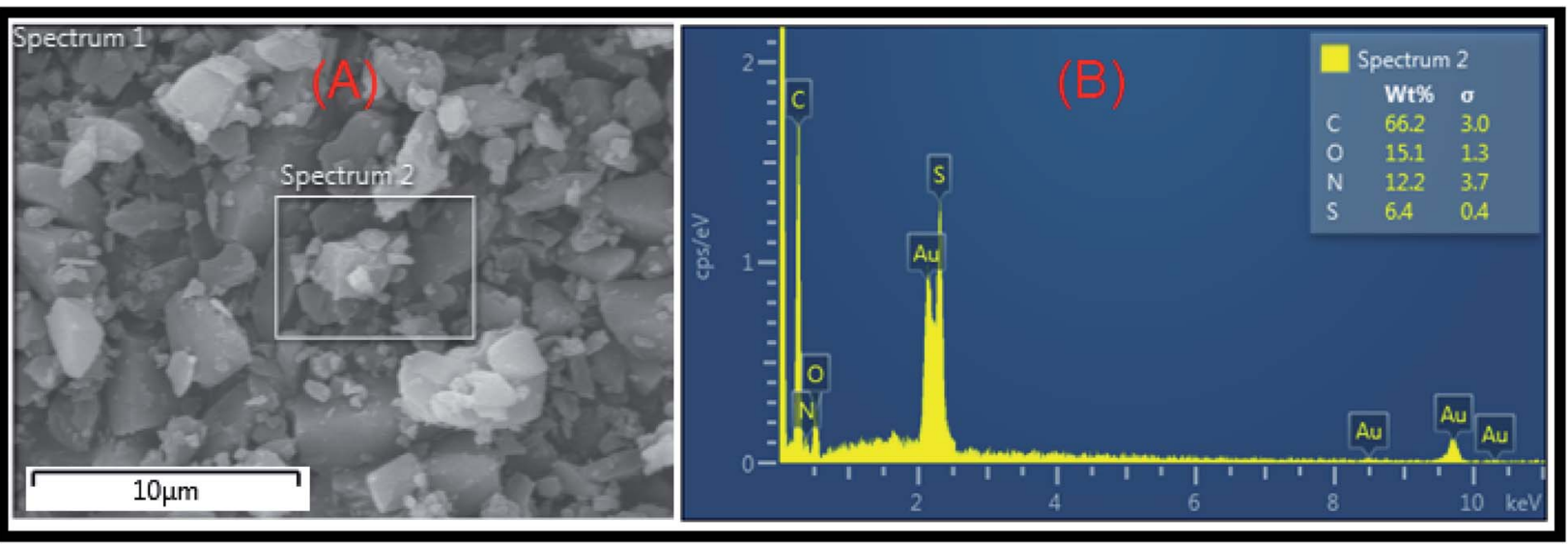

Fig. 4 SEM (A), and EDX spectra (B) of BSB. 


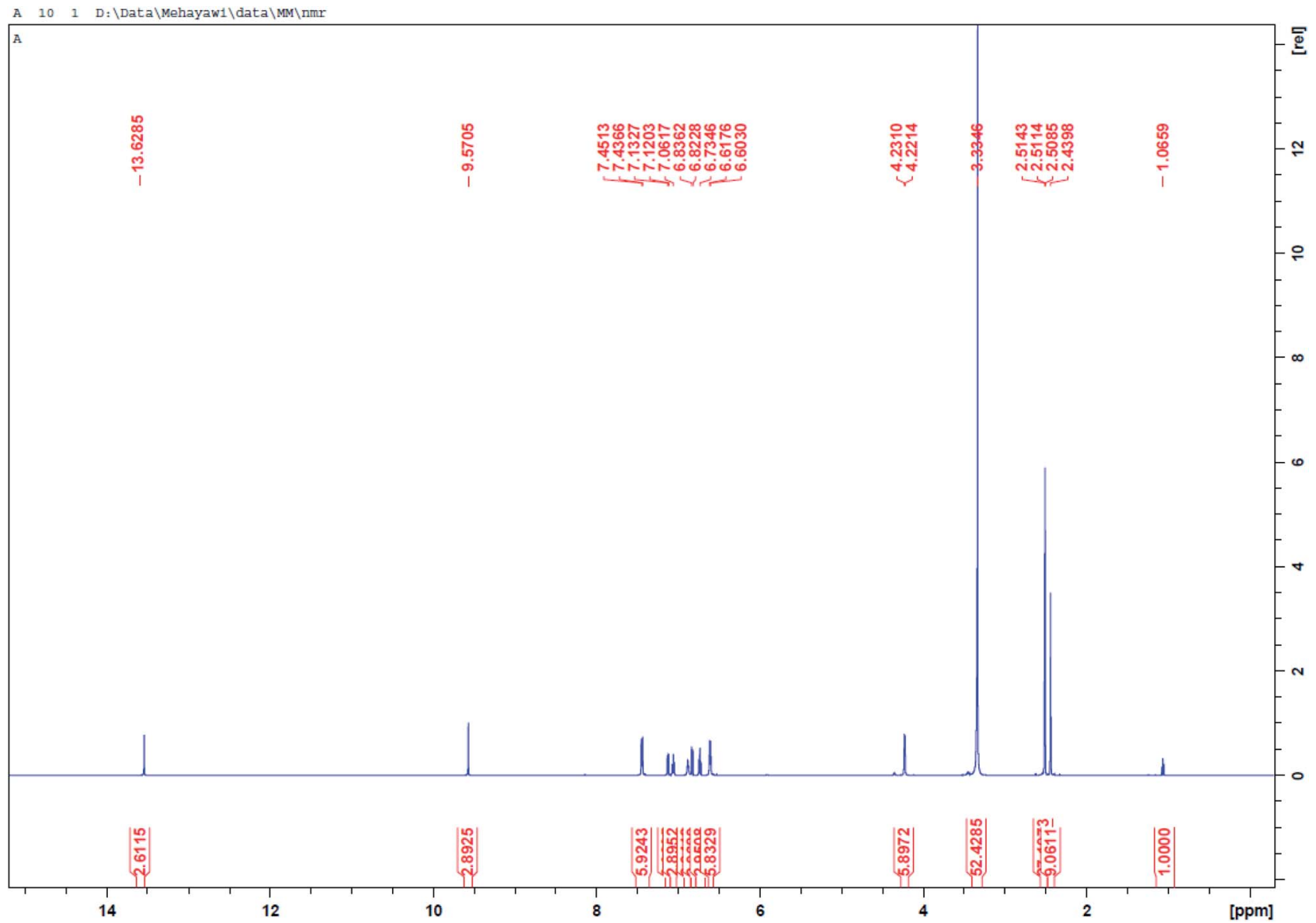

Fig. 5 The ${ }^{1} \mathrm{H}-\mathrm{NMR}$ spectrum of BSP.

sulfonamide group showed a peak at $13.62 \mathrm{ppm}$, while the signal of the phenolic proton of BSP appeared at a frequency of $9.57 \mathrm{ppm} .{ }^{31}$ The signals of the aromatic protons displayed as multiplet between $6.60 \mathrm{ppm}$ and $7.45 \mathrm{ppm}$. The methyl group protons attached to the thiazole ring appeared as a singlet peak at 2.51. The ${ }^{13} \mathrm{C}$-NMR spectrum (Fig. 6) revealed the carbon centers contained in the thiazole ring at $167.61 \mathrm{ppm}$, and $152.67 \mathrm{ppm}$, respectively. ${ }^{28}$ While the carbon atom bearing the hydroxyl group in the phenolic ring $(\mathrm{C}-\mathrm{OH})$ appeared at $155.71 \mathrm{ppm}^{31}$

Then, the last step in which the BSB inhibitor was obtained involved a ring formation process from the interaction between the phenolic hydroxyl and the primary amine groups of the BSP in the presence of 1 equivalent quantity of formaldehyde molecules in dioxane at refluxing condition for 18 hours. ${ }^{32}$ The mechanism of the ring closure is depicted in Fig. 2b. That reaction pathway was adopted to avoid the reaction complexity encountered by the traditional approach for the synthesis of benzoxazines, which involves the reaction of phenols, formaldehyde, and primary amines in solvent and solventless systems. If that approach were used, there is the possibility of the interaction between the thiazole ring and the amine within the sulfamethizole structure and the target product may not be formed. ${ }^{33}$ The BSB structure was also examined using the FTIR, and NMR $\left({ }^{1} \mathrm{H} \&{ }^{13} \mathrm{C}\right)$, respectively, as presented in Fig. 3(ii) and 7, 8, respectively. The FTIR spectrum of BSB confirmed the synthesis of the compound by showing some of its unique structural features. The strong band at $3342 \mathrm{~cm}^{-1}$ is attributed to the $\mathrm{N}-\mathrm{H}$ vibration, and the oxazine ring stretch appeared at $947 \mathrm{~cm}^{-1}$ after the successful formation of the ring. The bands of the trisubstituted aromatic ring, and the oxazine (C-O-C asymmetric stretch) appeared around 1501 and $1272 \mathrm{~cm}^{-1}$, respectively. ${ }^{34}$ The ${ }^{1} \mathrm{H}$-NMR of BSB is presented in Fig. 3. It displayed the protons make-up of the BSB structure. The signal of the protons attached to the oxazine ring $\left(\mathrm{O}-\mathrm{CH}_{2}-\mathrm{N}\right.$ and $\mathrm{Ar}-\mathrm{CH}_{2}-$ $\mathrm{N}$ ), appeared at frequencies of 5.89 , and $4.75 \mathrm{ppm}$, respectively. ${ }^{35}$ The ${ }^{13} \mathrm{C}-\mathrm{NMR}$ spectra of BSB further confirmed the successful synthesis of benzoxazine with the appearance of the two carbons of oxazine ring $\left(\mathrm{O}-\mathrm{CH}_{2}-\mathrm{N}\right.$ and $\left.\mathrm{Ar}-\mathrm{CH}_{2}-\mathrm{N}\right)$, at 58.56, and $50.58 \mathrm{ppm}$, respectively. ${ }^{30,36}$ The distribution of the elements on the surface of BSB was analyzed and quantified by EDX Fig. 4 . The surface on which this elemental analysis was performed is presented in Fig. 4A, while the EDX spectrum is shown in Fig. 4B. The EDX spectrum shows peaks due to elements such as carbon (C), nitrogen (N), oxygen (O), and sulphur (S), with the compositions of $66.2,15.1,12.2$ and $6.4 \%$, respectively. These are elemental makeup of BSB, and this further confirmed the synthesis of this novel hetero-cyclic compound. 


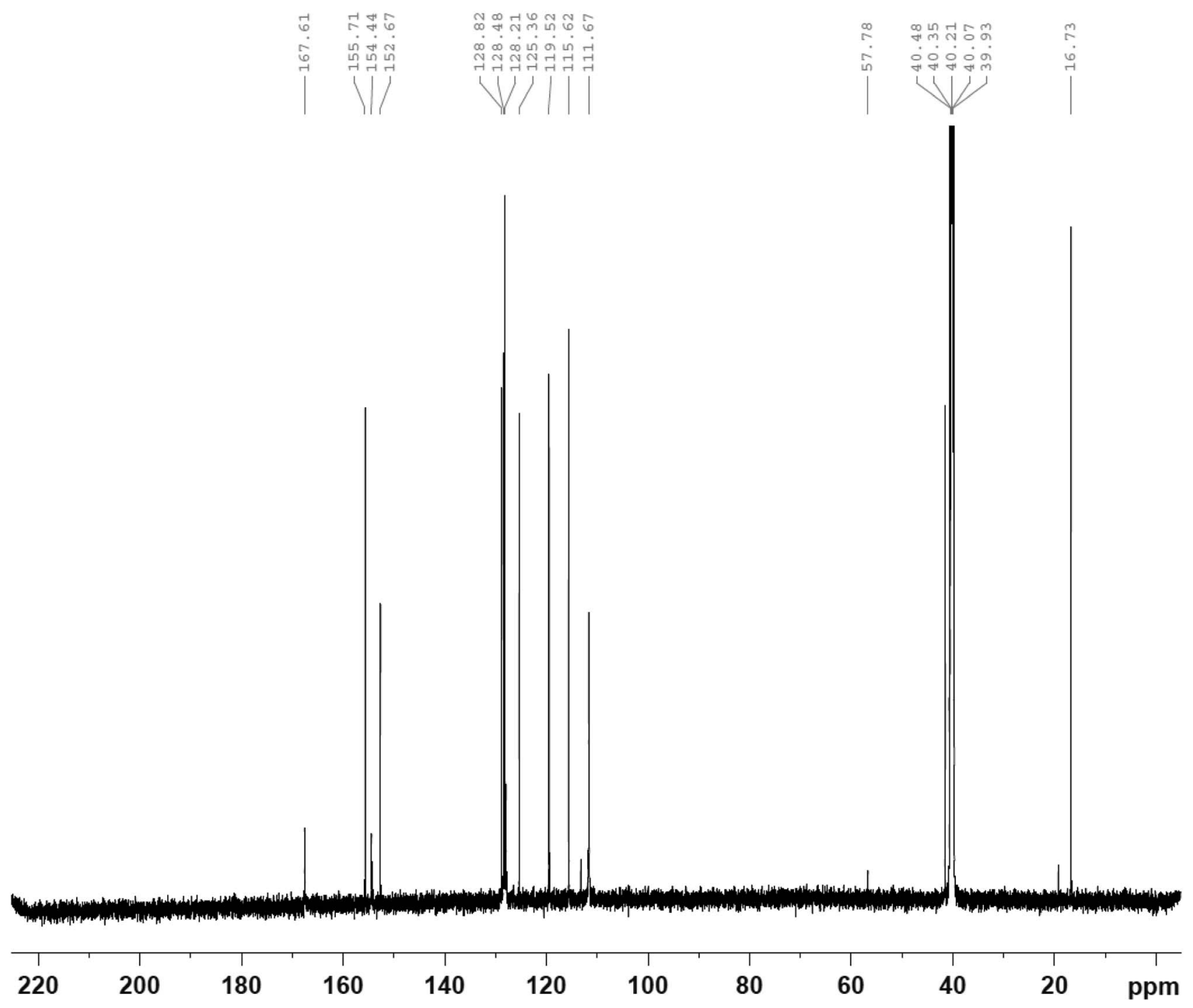

Fig. 6 The ${ }^{13} \mathrm{C}-\mathrm{NMR}$ spectrum of BSB.

\subsection{Weight loss}

Weight loss analysis was conducted at room temperature $(25 \pm$ 1), 40 and $60^{\circ} \mathrm{C}$, and the outcome is presented in Table 1 . The inhibited test solutions significantly expressed lower corrosion rate values in comparison to the blank, and the corrosion rate decreases with increase in BSB concentration. Regarding the carbon steel, the corrosion rate decreases from $3.849 \mathrm{~mm}$ per year in the blank to $2.535 \mathrm{~mm}$ year in $100 \mathrm{ppm}$ of BSB and to $0.572 \mathrm{~mm}$ per year in $400 \mathrm{ppm}$ of BSB. The adsorption of the BSB molecules onto the surface of the substrate to form a protective BSB film on the substrate surface is responsible for the observed reduction in the rate of corrosion. This film blocks the corrosive species from reaching the surface of steel. The observed higher inhibition efficiency (Table 1) with increasing BSB concentration is consequence of increasing amount of adsorbed BSB molecules on the steel surface. The highest concentration $(600 \mathrm{ppm})$ exhibited the optimum inhibition efficiency of over $88 \%$.
Furthermore, the effect of varying medium temperature on the inhibition performance of the BSB inhibitor was tested using; a blank acidic solution, and an acidic solution containing $600 \mathrm{ppm}$ of $\mathrm{BSB}$, at 40 and $60^{\circ} \mathrm{C}$, respectively. The corrosion rate of metals generally increases as the temperature of the corrosive environment rises. The effect is due to the increased average kinetic energy of the corrosive elements. ${ }^{37}$ The result revealed how the corrosion rate increased significantly for the blank solution from $3.849 \mathrm{~mm}$ per year at room temperature to 10.28 at $40{ }^{\circ} \mathrm{C}$ (an increment in the corrosion rate of over $267 \%$ ), and to $53.24 \mathrm{~mm}$ per year at $60{ }^{\circ} \mathrm{C}$ (an increment in the corrosion rate of over $517 \%$ ). Contrarily, the increase in the corrosion rate for the BSB inhibited solution with increasing temperature was not significant in comparison with values obtained for the blank. The rate increased from $0.448 \mathrm{~mm}$ per year at room temperature to 0.974 and $4.561 \mathrm{~mm}$ per year at 40 (increment in corrosion rate of over $217 \%$ ) and $60{ }^{\circ} \mathrm{C}$ (an increment in the rate of over $468 \%$ ) respectively. The slight increase in inhibition efficiency with temperature at the 600 ppm BSB concentration 


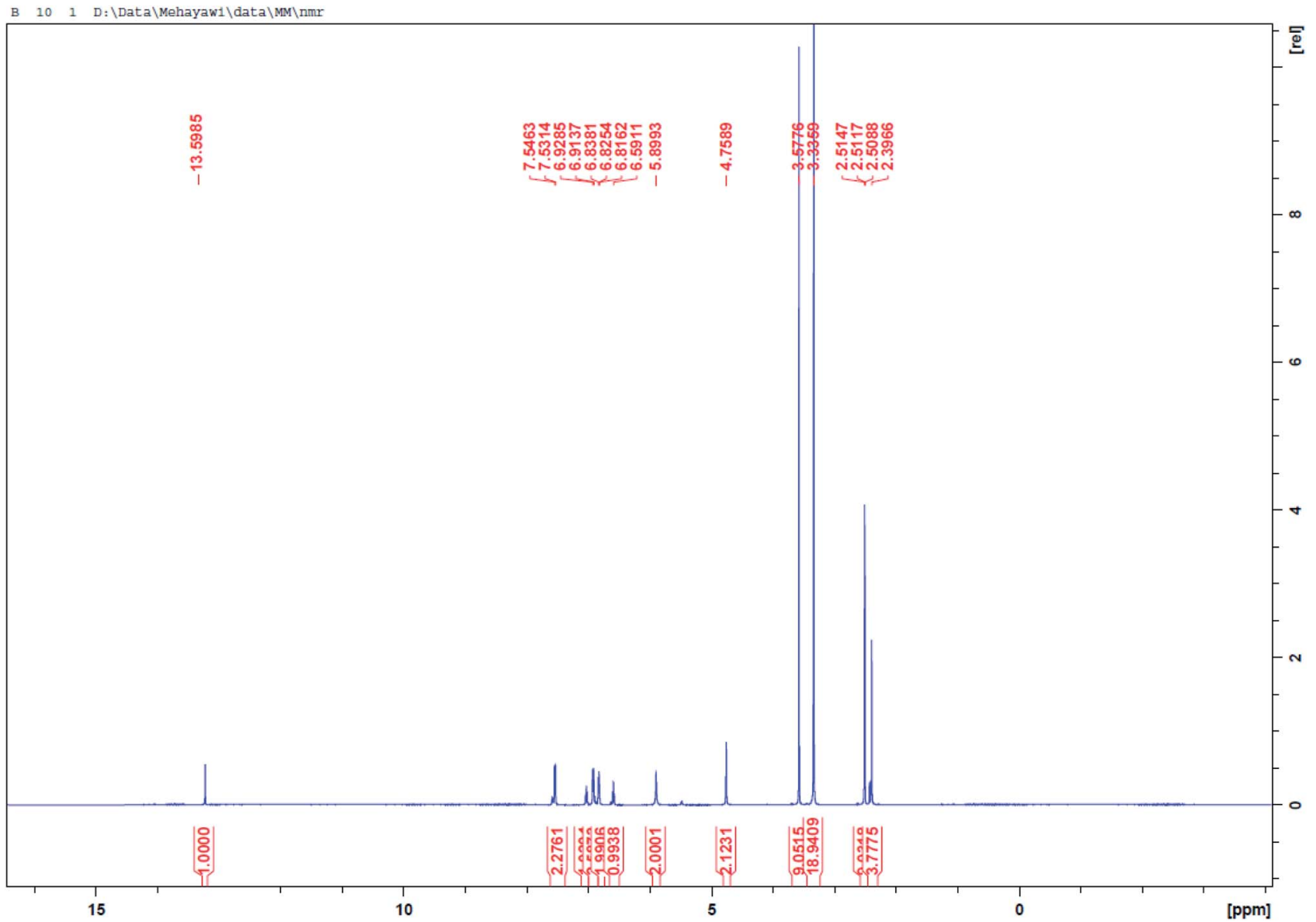

Fig. 7 The ${ }^{1} \mathrm{H}-\mathrm{NMR}$ spectrum of BSB.

(Table 1) suggests BSB adsorption at this concentration could involve the chemisorption adsorption mechanism. In the chemisorption mechanism (which involves charge sharing or charge transfer from inhibitor molecules to metal surface), the force of attraction between inhibitor molecules are very strong much stronger than the physisorption van der Waals forces of interaction. Therefore, the inhibition efficiency is significantly increases from lower BSB concentration to higher concentration. The corrosion rate in the presence of BSB inhibitor at $60{ }^{\circ} \mathrm{C}$ is much lower than the maximum acceptable corrosion rate $(50.80 \mathrm{~mm}$ per year) for high temperature acidizing environments. ${ }^{38}$

\subsection{Electrochemical measurement techniques}

3.3.1. EIS technique. The electrochemical examination of the BSB inhibited solutions, and the blank sample, was analyzed, and the results are presented as Nyquist plot, Bode phase plot, and Bode modulus curves, respectively (Fig. 9). The shape of the Nyquist plot obtained from using a blank sample solution, and the inhibitor solution, can be described as depressed, semi-circular capacitive arcs. The effect resulted from imperfections due to causes such as the existence of impurity on the electrode, electrode surface in homogeneity, and roughness. ${ }^{30}$ The arcs correspond to the two-time constants in the Bode graphs at high and low frequency zones. At the high frequency zone, the time-constant $\left(R_{\mathrm{f}} / / \mathrm{CPE}_{\mathrm{f}}\right)$ represents the formed corrosion product/BSB film, while the double layer formed at the interface of the metal/electrolyte relates to the time constant $\left(\mathrm{R}_{\mathrm{ct}} / / \mathrm{CPE}_{\mathrm{dl}}\right)$ at the low frequencies zone. The resemblance in the individual curve shapes for BSB inhibited and uninhibited solutions is the evidence in support of the fact that the inhibitor operational process does not change the corrosion mechanism. However, the BSB inhibited solutions exhibited a bigger Nyquist plot diameter than the blank solution, and the Nyquist plot diameter for the inhibited solution increased as the BSB concentration also increased (Fig. 9a). That behavior suggested a higher charge transfer resistance $\left(R_{\mathrm{ct}}\right)$ for the inhibited solution which implied it exhibited a lower rate of corrosion. The inhibitor caused a lower rate of corrosion due to the formation of a BSB adsorbed layer on the steel surface. The barrier prevented the steel from the attacks of the corrosive acid, and the increased concentration of the BSB caused more coverage on the surface of the steel. It was the phenomenon that translated into the lowering of the corrosion rate with increased BSB concentration.

The Bode phase plots display two distinguishable peaks for the inhibited and the blank sample solutions which correspond to two-time constants (Fig. 9b). From the Bode plots, the phase angles showed peak heights that are higher for the solutions 


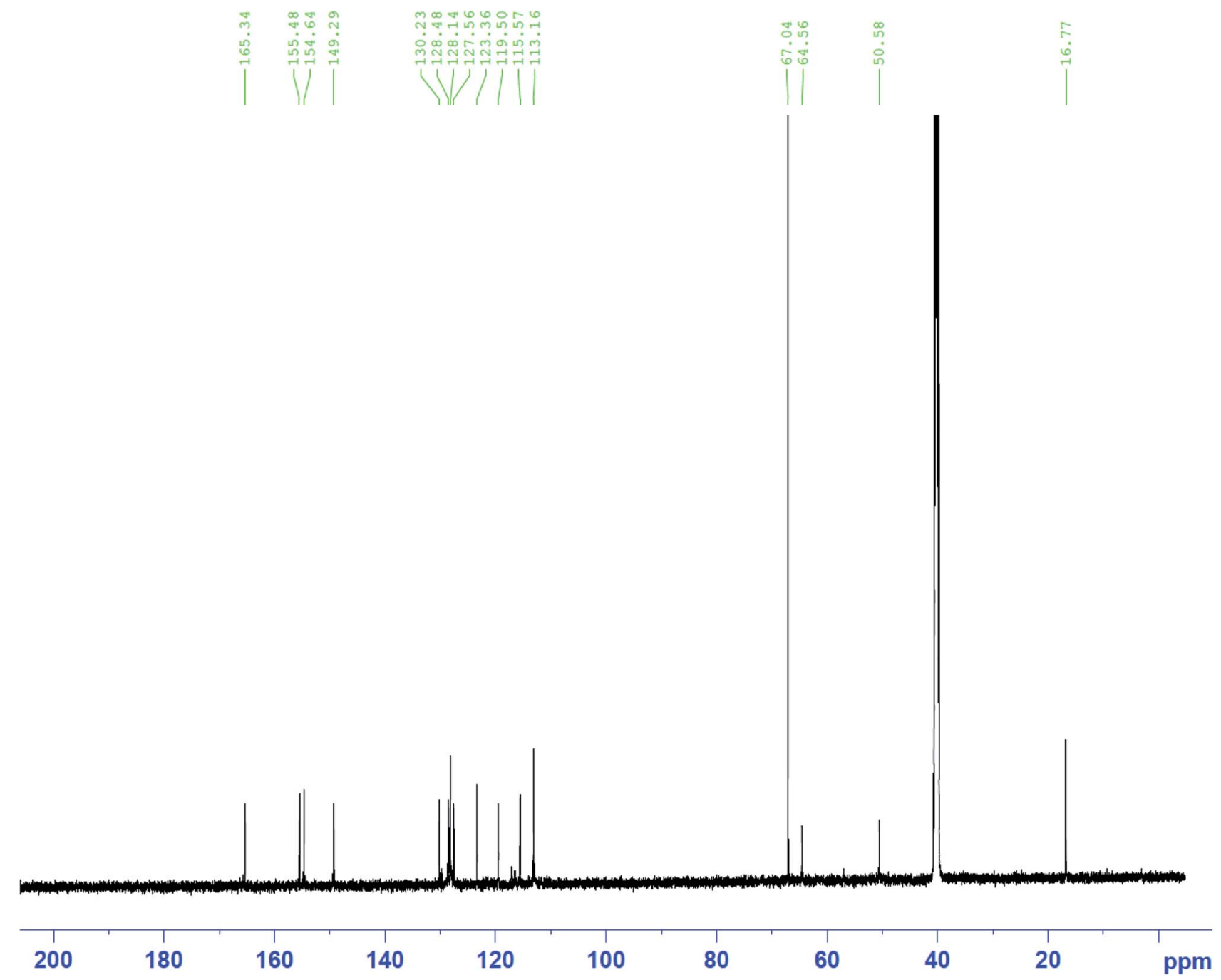

Fig. 8 The ${ }^{13} \mathrm{C}-\mathrm{NMR}$ spectrum of BSB.

containing the BSB compound, than for the blank solution, and these heights increased with more BSB concentration. At the intermediate frequencies in the Bode modulus plots (where it appeared linear) (Fig. 9c), this linearity is even more for the solutions containing the inhibitor than the one with the blank solution. That trend signified the lowering rate of corrosion by the inhibitor, and the corrosion rate also decreased with higher concentrations of the inhibitor. ${ }^{39}$

The EIS spectra were fitted using an equivalent circuit (Fig. 9d) that comprised of the following elements: the $\mathrm{CPE}_{\mathrm{f}}$

Table 1 Weight loss measurement parameters for blank and BSB inhibited solutions at room temperature $\left(25 \pm 1^{\circ} \mathrm{C}\right) 40$ and $60{ }^{\circ} \mathrm{C}$

\begin{tabular}{|c|c|c|c|c|}
\hline $\begin{array}{l}\text { Temperature } \\
\left({ }^{\circ} \mathrm{C}\right)\end{array}$ & Concn. of BSB (ppm) & $\begin{array}{l}\text { Weight loss }(\mathrm{g}) \pm \\
\text { standard deviation }\end{array}$ & $\begin{array}{l}\text { Corrosion rate } \\
\text { (mm per year) }\end{array}$ & $\%$ IE \\
\hline \multirow[t]{4}{*}{$25 \pm 1$} & Blank & $0.249 \pm 0.0007$ & 3.849 & - \\
\hline & 200 & $0.100 \pm 0.0014$ & 1.546 & 59.83 \\
\hline & 300 & $0.078 \pm 0.0042$ & 1.206 & 68.67 \\
\hline & 400 & $0.037 \pm 0.0007$ & 0.572 & 85.14 \\
\hline \multirow[t]{2}{*}{40} & Blank & $0.665 \pm 0.0590$ & 10.28 & - \\
\hline & 600 & $0.063 \pm 0.0000$ & 0.974 & 90.53 \\
\hline \multirow[t]{2}{*}{60} & Blank & $3.444 \pm 0.1062$ & 53.24 & - \\
\hline & 600 & $0.295 \pm 0.0120$ & 4.561 & 91.43 \\
\hline
\end{tabular}



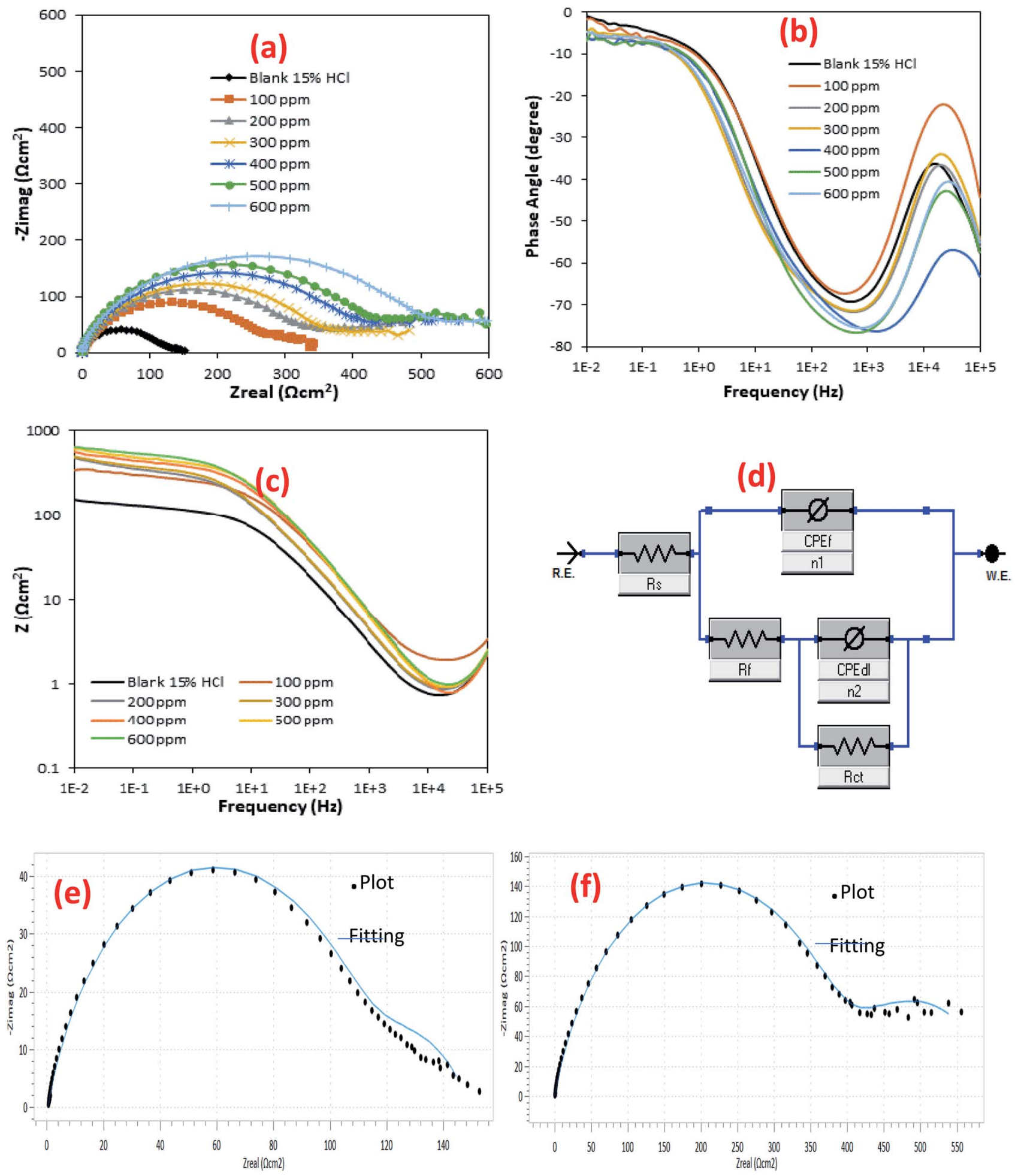

Fig. 9 (a) Nyquist, (b) Bode phase (c) Bode modulus plots recorded for the blank and BSB inhibited solutions (d) equivalent circuit used for fitting the test solutions impedance curves (e) and (f) fitted Nyquist plots for blank and 400 ppm BSB inhibited solutions.

(film constant phase element), $R_{\mathrm{S}}$ (solution resistance), $n_{1}$ (phase shift 1), $R_{\mathrm{ct}}$ (charge transfer resistance), $R_{\mathrm{f}}$ (film resistance), $n_{2}$ (phase shift 2), \& $\mathrm{CPE}_{\mathrm{dl}}$ (double layer constant phase element). The EIS elements have quantities obtained after the EIS spectra fitting, which are listed in Table 2, while the $\%$ efficiency of the inhibitor (\%IE) was computed using eqn (3).

$$
\% \mathrm{IE}_{\mathrm{EIS}}=\left(1-\frac{R_{\mathrm{p}}^{\mathrm{o}}}{R_{\mathrm{p}}^{\mathrm{I}}}\right) \times 100
$$


Table 2 Electrochemical impedance spectroscopy measurement parameters for the blank and BSB inhibited solutions at room temperature (25 $\left.\pm 1^{\circ} \mathrm{C}\right)$

\begin{tabular}{|c|c|c|c|c|c|c|c|c|c|c|c|c|}
\hline \multirow{2}{*}{$\begin{array}{l}\text { Concn. of } \\
\text { metformin } \\
(\mathrm{ppm})\end{array}$} & \multirow[b]{2}{*}{$\begin{array}{l}R_{\mathrm{S}}(\Omega \\
\left.\mathrm{cm}^{2}\right)\end{array}$} & \multicolumn{2}{|l|}{$\mathrm{CPE}_{\mathrm{f}}$} & \multirow[b]{2}{*}{$\begin{array}{l}R_{\mathrm{f}}(\Omega \\
\left.\mathrm{cm}^{2}\right)\end{array}$} & \multirow[b]{2}{*}{$\begin{array}{l}C_{\mathrm{f}}(\mu \mathrm{F} \\
\left.\mathrm{cm}^{-2}\right)\end{array}$} & \multicolumn{2}{|l|}{$\mathrm{CPE}_{\mathrm{dl}}$} & \multirow[b]{2}{*}{$\begin{array}{l}R_{\mathrm{ct}}(\Omega \\
\left.\mathrm{cm}^{2}\right)\end{array}$} & \multirow[b]{2}{*}{$\begin{array}{l}C_{\mathrm{dl}}(\mu \mathrm{F} \\
\left.\mathrm{cm}^{-2}\right)\end{array}$} & \multirow[b]{2}{*}{$\begin{array}{l}R_{\mathrm{p}}(\Omega \\
\left.\mathrm{cm}^{2}\right)\end{array}$} & \multirow[b]{2}{*}{$\% \mathrm{IE}$} & \multirow[b]{2}{*}{$\begin{array}{l}\chi^{2} \times \\
10^{-3}\end{array}$} \\
\hline & & $\begin{array}{l}Y_{\mathrm{o}_{1}}\left(\mathrm{~m} \Omega \mathrm{s}^{n}\right. \\
\left.\mathrm{cm}^{-2}\right)\end{array}$ & $n_{1}$ & & & $\begin{array}{l}Y_{\mathrm{O}_{2}}\left(\mathrm{~m} \Omega \mathrm{s}^{n}\right. \\
\left.\mathrm{cm}^{-2}\right)\end{array}$ & $n_{2}$ & & & & & \\
\hline Blank & 0.253 & 33.49 & 0.646 & 35.85 & 443.9 & 0.335 & 0.785 & 112.9 & 141.0 & 146.8 & - & 0.072 \\
\hline 100 & 0.515 & 17.80 & 0.720 & 84.41 & 188.5 & 0.164 & 0.762 & 260.1 & 61.19 & 344.5 & 57.39 & 0.040 \\
\hline 200 & 0.147 & 23.39 & 0.753 & 157.1 & 254.5 & 0.252 & 0.758 & 328.6 & 121.7 & 485.7 & 69.78 & 0.144 \\
\hline 400 & 0.760 & 14.98 & 0.625 & 217.6 & 115.9 & 0.147 & 0.772 & 395.2 & 63.83 & 612.6 & 76.04 & 0.028 \\
\hline 500 & 0.867 & 12.08 & 0.623 & 245.2 & 102.9 & 0.114 & 0.810 & 413.0 & 61.08 & 658.2 & 77.70 & 0.041 \\
\hline 600 & 1.268 & 19.32 & 0.652 & 179.9 & 176.5 & 0.154 & 0.755 & 499.8 & 63.54 & 679.7 & 78.40 & 0.011 \\
\hline
\end{tabular}

where: $R_{\mathrm{p}}$ is a sum of $R_{\mathrm{f}} \& R_{\mathrm{ct}}$ and $R_{\mathrm{p}}^{\mathrm{o}}$ represents the resistance of zero inhibitor solution while $R_{\mathrm{p}}^{\mathrm{I}}$ is for the resistance of inhibitor solution.

By virtue of the depressed character of the capacitive loop, the CPE rather than a capacitor is used to described the formed double layer and can be computed using eqn (4). ${ }^{40}$

$$
Z_{\mathrm{CPE}}=Y_{\mathrm{o}}^{-1}(j \omega)^{-n}
$$

The components; $Y_{\mathrm{o}}, \omega, n$, and $j$, are the CPE magnitude, angular frequency, phase shift \& the square root of -1 , respectively.

The high resistance to electrochemical corrosion exhibited by the inhibited samples solutions relative to that of the blank sample is expressed by the values of the resistances $\left(R_{\mathrm{f}} \& R_{\mathrm{ct}}\right)$ presented in Table 2 . The results from all these parameters are significantly higher for the inhibited solution than the blank samples. That is linked to the effect of the formed BSB preventive layers formed on the surface of the steel. The surface is covered and prevented from attack by the harsh acid, which caused a reduction in the mass and charge transfer. The $R_{\mathrm{f}}$ and $R_{\mathrm{ct}}$ parameters expressed higher results for the inhibited solution than the blank sample due to the formation of a surface with lesser porosity, and higher resistance to charge transfer for the protected steel surface. The porosity of the protective layer on the steel surface is expressed in terms of $Y_{\mathrm{o}}$ quantity. The solutions containing inhibitor showed relatively smaller values for $Y_{\mathrm{o}}$ in comparison to the blank solution. That indicated a steel surface with lesser porosity, due to the protective layers of the BSB inhibitor.
3.3.2. LPR technique. The LPR technique employs a small polarization of $\pm 10 \mathrm{mV}$ from OCP to allow for a direct correlation between corrosion potential and current, thus making the LPR technique a non-destructive one. The values of the parameters obtained from fitting the LPR are presented in Table 3. No defined trend was observed in $E_{\text {corr }}$ values for the inhibited solutions and these values are more inclined towards the negative direction in comparison to the blank solution. The corrosion rate significantly decreased in the presence of the BSB inhibitor, and this rate decreased as the concentration of the BSB becomes higher. Also, much greater polarization resistances were observed for the inhibited solutions in comparison to the uninhibited solution. That is an indication of higher resistance to corrosion in the presence of the BSB inhibitor. Eqn (5) was employed to calculate BSB percentage inhibition efficiency (\%IE).

$$
\% \mathrm{IE}_{\mathrm{LPR}}=\left(1-\frac{R_{\mathrm{p}}^{\mathrm{o}}}{R_{\mathrm{p}}^{\mathrm{I}}}\right)
$$

where, $R_{\mathrm{p}}^{\mathrm{o}}$ denotes the polarisation resistance of the blank and $R_{\mathrm{p}}^{\mathrm{I}}$ denotes the polarization resistance of the inhibited solutions.

The calculated inhibition efficiencies are in conformity with findings from both weight loss and EIS measurements. The computation reveals higher efficiencies resulting from increased BSB concentration that caused higher amount of the adsorbed BSB film on the steel substrate.

3.3.3. PDP technique. The PDP, unlike the LPR technique, uses a broader polarization, which is usually within the range of

Table 3 Potentiodynamic polarization and linear polarization resistance measurements parameters for the blank and BSB inhibited solutions at

\begin{tabular}{|c|c|c|c|c|c|c|c|c|c|c|}
\hline $\begin{array}{l}\text { Concn. of metformin } \\
\text { (ppm) }\end{array}$ & \multicolumn{6}{|l|}{ PDP method } & \multicolumn{4}{|l|}{ LPR } \\
\hline Blank & -390.3 & 167.9 & 100.7 & 160.5 & 1.948 & - & -380.9 & 2.052 & 147.3 & - \\
\hline 100 & -375.1 & 50.87 & 77.62 & 156.4 & 0.590 & 69.71 & -370.9 & 0.974 & 310.6 & 52.58 \\
\hline 200 & -336.0 & 28.11 & 85.76 & 123.3 & 0.326 & 83.26 & -332.5 & 0.613 & 493.3 & 70.14 \\
\hline 500 & -373.8 & 21.03 & 111.9 & 112.7 & 0.244 & 87.47 & -357.0 & 0.477 & 633.8 & 76.76 \\
\hline 600 & -335.7 & 19.61 & 68.94 & 121.9 & 0.228 & 88.30 & -334.0 & 0.458 & 660.5 & 77.70 \\
\hline
\end{tabular}
room temperature $\left(25 \pm 1^{\circ} \mathrm{C}\right)$ 


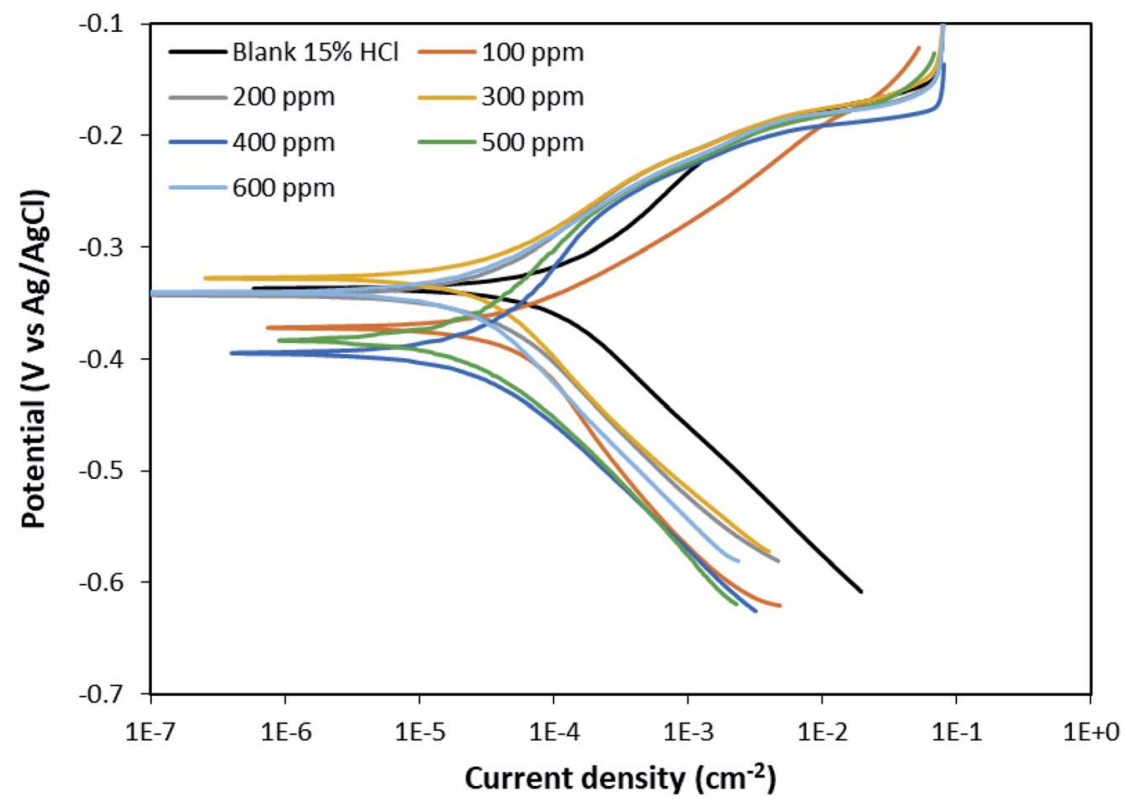

Fig. 10 Potentiodynamic polarization plots recorded for the blank and BSB inhibited solutions.

200 to $400 \mathrm{mV}$ from OCP, hence, making it a destructive one. Nevertheless, it gives more detailed information than the LPR technique, such as the consequence of inhibitor on the dissolution of metal at the anode with the liberation of $\mathrm{H}_{2}$ gas from the cathode. The PDP graphs for the BSB inhibited solution, and blank samples, are shown in Fig. 10. The plots showed BSB inhibitor to have a higher effect on the cathodic current density, which suggests that BSB acts predominantly as a cathodic-type indicator. The corrosion potentials $\left(E_{\text {corr }}\right)$ values exhibited in

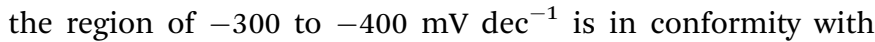
previously reported studies. ${ }^{37}$ The $E_{\text {corr }}$ values for the inhibited solutions exhibited a mixed behavior with majority slightly shifted less negative than that of the blank. The $\beta_{\mathrm{a}}$ and $\beta_{\mathrm{c}}$ values also exhibited mixed behaviors with the $\beta_{\mathrm{c}}$ having a higher range of $63.14 \mathrm{mV} \mathrm{dec}^{-1}$ than $\beta_{\mathrm{a}}$ with a range of $42.94 \mathrm{mV} \mathrm{dec}^{-1}$ these confirms BSB to act as a mixed-type corrosion inhibitor with cathodic predominance. ${ }^{40}$

Table 3 gives the values of the PDP parameters after fitting the plots. The inhibition efficiency of the BSB inhibitor was calculated using eqn (6).

$$
\mathrm{IE}(\mathrm{PDP}) \%=\left(1-\frac{I_{\text {corr }}^{\mathrm{I}}}{I_{\text {corr }}^{\mathrm{o}}}\right)
$$

where, denotes the $I_{\text {corr }}^{\mathrm{o}}$ denotes the corrosion current density for the blank solution while $I_{\text {corr }}^{\mathrm{I}}$ denotes the corrosion current density for the inhibited solution.

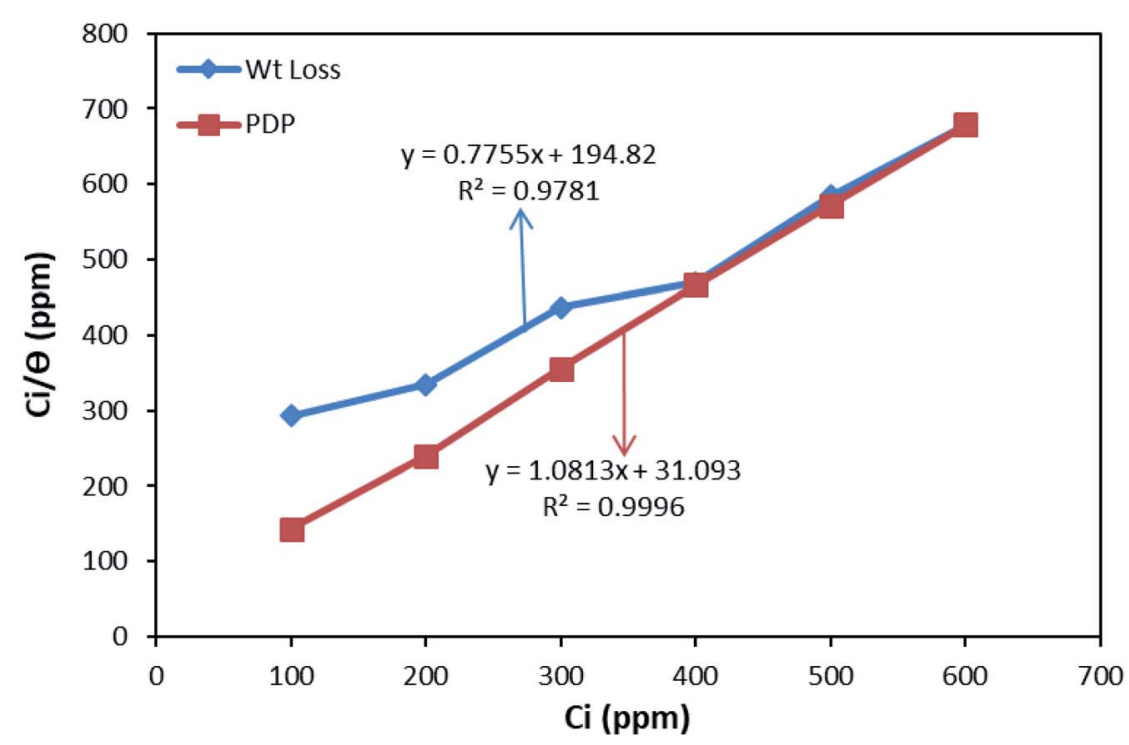

Fig. 11 Langmuir adsorption isotherms for adsorption of BSB on the carbon steel surface in $15 \% \mathrm{HCl}$. 
Table 4 Langmuir adsorption parameters for BSB adsorption to carbon steel surface in $15 \% \mathrm{HCl}$ estimated from the weight loss and PDP experimental data

\begin{tabular}{lllll}
\hline Technique & $\Delta G_{\text {ads }}^{\circ}\left(\mathrm{kJ} \mathrm{mol}^{-1}\right)$ & $K_{\text {ads }}\left(\mathrm{ppm}^{-1}\right)$ & Slope & $R^{2}$ \\
\hline Weight loss & -21.178 & $5.133 \times 10^{-3}$ & 0.776 & 0.978 \\
PDP & -25.726 & $32.16 \times 10^{-3}$ & 1.081 & 0.999
\end{tabular}

The solutions inhibited by the presence of BSB showed lower values of the corrosion current density $\left(I_{\text {corr }}\right)$ compared to the blank sample, which suggests a significant decrease in the corrosion rate with the presence of the BSB inhibitor. The existence of the BSB prevented the ions of the corrosive medium from reaching the metal surface. This argument is reflected in the lower $I_{\text {corr }}$ quantities for the solutions of the BSB, and the results here also agree with the outcomes from the weight loss, EIS and LPR experiments.

\subsection{Adsorption isotherm}

Inhibitors of corrosion generally form single or multiple layers, which serve as a barrier on metal surfaces and hence block it from attack. The mechanism for the formation of the layers could be through the adsorption of the inhibitor molecules on the metal surface via physical, chemical interaction, or both processes. The data from the weight loss analysis, and PDP measurements, were used to understand the mode of adsorption of BSB. Several adsorption isotherms (models), such as the Langmuir, Freundlich, and Tempkin, were analyzed. It was, however, found that the best fit was obtained by using
Langmuir adsorption isotherm. The isotherm assumes a monolayer adsorbate molecules adsorption that is homogeneous and independent, ${ }^{\mathbf{4 1}}$ which is expressed as:

$$
\frac{C_{\mathrm{i}}}{\theta}=\frac{1}{K_{\mathrm{ads}}}+\frac{a_{\mathrm{L}}}{K_{\mathrm{L}}} C_{\mathrm{i}}
$$

where: $C_{\mathrm{i}}$ is concentration of $\mathrm{BSB}, \theta$ is extent of surface coverage, $K_{\text {ads }}$ is equilibrium constant for adsorption process $(\theta$ evaluated with respect to $\% \mathrm{IE} / 100$, assuming a correlation between the $\%$ IE and the extent of surface coverage) and $K_{\mathrm{L}} \& a_{\mathrm{L}}$ are the Langmuir isotherm constants.

The graph of $C_{\mathrm{i}} / \theta$ against $C_{\mathrm{i}}$ gives a linear shape with $a_{\mathrm{L}} / K_{\mathrm{L}}$ as the slope and $1 / K_{\mathrm{ads}}$ as the intercept. The theoretical monolayer capacity is numerically obtained as the slope, $a_{\mathrm{L}} / K_{\mathrm{L}} \cdot{ }^{\mathbf{4 1}}$ The values of correlation coefficient $\left(R^{2}\right): 0.9781$ and 0.9996 for the weight loss and PDP data, are gotten from the straight-line graphs (Fig. 11), and this is an indication that the data followed the Langmuir isotherm theory. The values of the components obtained from the plots are listed in Table 4.

$K_{\text {ads }}$ is said to be related to the Gibbs free energy of adsorption $\left(\Delta G_{\text {ads }}^{\circ}\right)$ as expressed in eqn (8). ${ }^{37,42}$

$$
\Delta G_{\mathrm{ad}}^{\circ}=-R T \ln \left(1 \times 10^{6} K_{\mathrm{ads}}\right)
$$

where: $R$ is molar gas constant $\left(8.314 \mathrm{~J} \mathrm{~K}^{-1} \mathrm{~mol}^{-1}\right) \& T$ is temperature (in Kelvin).

The values of $\Delta G_{\text {ads }}^{\circ}$ round $-20 \mathrm{~kJ} \mathrm{~mol}^{-1}$ are said to be associated with the physisorption mechanism. This mechanism is a bonding process between molecules of the inhibitor and the atoms of the metal residing on the surface, while quantities of $\Delta G_{\text {ads }}^{\circ} \geq-40 \mathrm{~kJ} \mathrm{~mol}^{-1}$ are associated with the chemisorption mechanism. The chemisorption mechanism involves the
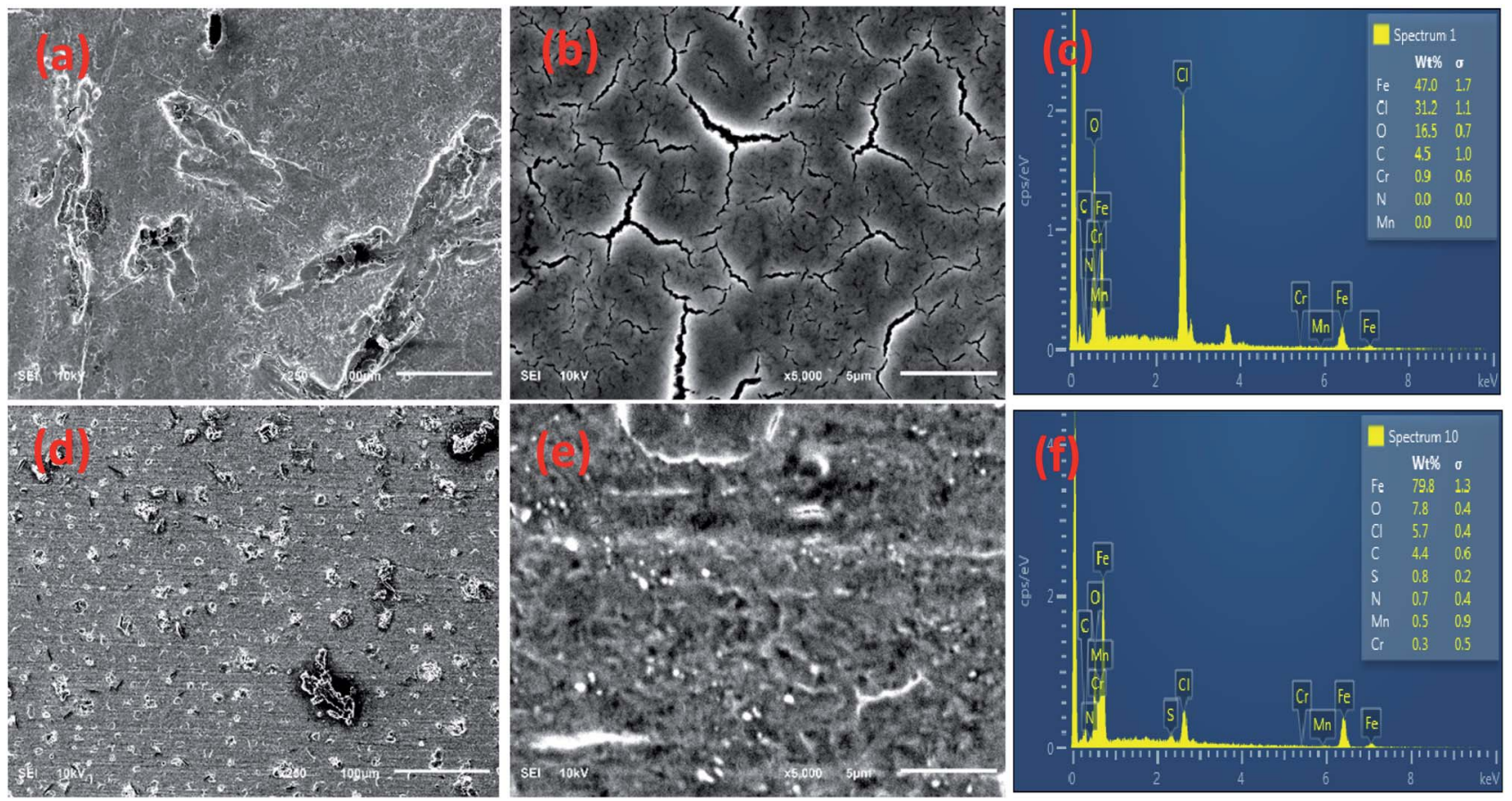

Fig. 12 (a) Low, (b) high SEM image \& (c) EDX spectrum of carbon steel surface after 24 hours immersion in the blank solution (d) low, (e) high SEM image $\&$ (f) EDX spectrum of carbon steel surface after 24 hours immersion in 600 ppm BSB inhibited solution. 



Fig. 13 2D, 3D AFM images \& roughness parameters for the carbon steel surface after $24 \mathrm{~h}$ immersion in the (a) blank and (b) 600 ppm BSB inhibited solutions.

donation of electrons pair from the molecules of the inhibitor to the vacant d-orbitals of the metal, whereas values between -20 and $-40 \mathrm{~kJ} \mathrm{~mol}^{-1}$ suggests both mechanism. ${ }^{43}$ However, in this study, the values of -21.18 and $-25.73 \mathrm{~kJ} \mathrm{~mol}^{-1}$ were obtained using the data of the weight loss and PDP analyses, suggesting the adsorption of BSB to the steel surface to have occurred via 
both mechanisms, with predominance via the physisorption mechanism which is in agreement with the PDP measurement.

\subsection{Surface characterization and analysis}

The magnified SEM images (low \& high), of the surface of the steel substrates, after their immersion for 24 hours, in the solution of blank and the BSB solutions, are given in Fig. 12 . The unprotected substrate surface exhibits roughness with dips and cracks unevenly distributed over it (Fig. 12a and b). A consequence of the corrosion effect suffered by the substrate immersed in the blank solution. While a relatively smooth surface, with no cracks and dip, was exhibited by the inhibited steel surface. Also, the molecules of the BSB are distributed evenly over the entire steel surface (Fig. 12d and e). The protected steel surface (Fig. 12d) exhibits a more compact film that looks far less porous than the cracked, non-compact film shown by the unprotected steel surface. The much smoother surface exhibited by the BSB covered steel is a consequence of the adsorbed BSB molecules which cover and protect the steel surface against attack by the aggressive acid.

EDX spectra were collected to get the elemental composition of the formed film on surfaces of the unprotected and protected steel surface to gain insight into the adsorption phenomena of BSB. Fig. 12c and f contain the EDS spectra of the film formed on the surface of the substrate immersed in the blank, and BSB inhibitor solutions, respectively. The percentage weight of Fe in the film, found on the surface of the unprotected steel is $47.0 \%$. While the chloride and oxygen, contents are $31.2 \mathrm{wt} \%$ and $16.5 \mathrm{wt} \%$, respectively (Fig. 12c). That is a consequence of the aggressive chloride attack and the subsequent high Fe dissolution. A significantly lower chloride and oxygen contents of (5.7 wt\%) and (7.8 wt\%), respectively, with a much higher Fe content (79.8 wt\%), was exhibited by the formed BSB film (Fig. 12f). That is a consequence of the formation of a protective barrier by the adsorbed BSB molecules, which blocks the steel surface from attack by the corrosive chloride ions, thus preventing the steel from further dissolution. The presence of additional $\mathrm{N}$ and $\mathrm{S}$ peaks in the spectrum of the formed film and their absence in that of the unprotected steel surface is also evidence of BSB adsorption on the carbon steel surface. The SEM and EDS analyses confirmed the results obtained from Sections 3.2 and 3.3, which suggested the protection of the carbon steel against the aggressive acid attack by the adsorbed BSB film formed on its surface.

The formed films on the surfaces of the unprotected and protected steel were further analyzed by the AFM technique, which is a technique used to virtualize the surface of the materials in both two and three dimensions at the nanometer scale. The tool was employed to examine inhibitors effect on metal topography and surface roughness in different corrosive media. ${ }^{44-49}$ The 2D and 3D micrographs showing the films formed on the surfaces of the unprotected and protected steel are shown in Fig. 13. Some quantities, such as the values of the average roughness $\left(R_{\mathrm{a}}\right)$, the roughness root mean square $\left(R_{\mathrm{q}}\right)$, and the maximum profile valley depth $\left(R_{\mathrm{v}}\right)$, are given in Table 5 . The $R_{\mathrm{a}}$ and $R_{\mathrm{q}}$ indicate the height deviation, and the of surface height standard deviation, respectively.

The thin film on the surface of the unprotected steel expressed values of $R_{\mathrm{a}}, R_{\mathrm{q}} \& R_{\mathrm{v}}$ as $0.145,0.191 \& 0.645 \mu \mathrm{m}$, respectively. While the same parameters are given as 0.067 , $0.087 \& 0.243 \mu \mathrm{m}$, respectively, for the protected steel surface (Table 5). Higher values of these parameters for the unprotected steel relative the protected ones indicate porous steel surfaces. The values obtained proved the roughness of such surface as supported by the 2D and 3D micrographs images (Fig. 13a and b). The strong acid attack caused the creation of pores and roughness on the surface of steel immersed in the blank since no inhibitor is present. Hence, the adsorbed BSB inhibitor on the steel surface plays a key role in preventing the steel from the ions of corrosion. This argument is supported by previous results of the weight lost analysis, EIS, LPR, and PDP, respectively.

FTIR spectra were collected to understand the nature of the interaction of the BSB with the carbon steel surface, which will aid in proposing a mechanism for BSB adsorption. Comparative FTIR spectra of the pure BSB and the BSB film on the steel surface was used (Fig. 3). The formed BSB film shows similar vibrational bands with some slight shifts when compared to the spectrum of the pure BSB. That shows the adsorption of the BSB molecules onto the steel surface. In the BSB structure, the stretching vibrational bands of $\mathrm{C}-\mathrm{O}-\mathrm{C}$ (asymmetric), $\mathrm{S}=\mathrm{O}$ (symmetric), and $\mathrm{S}=\mathrm{O}$ (asymmetric) recorded at 3342, 1272, 1127 , and $1371 \mathrm{~cm}^{-1}$, for the native sample, were observed at 1289,1131 , and $1353 \mathrm{~cm}^{-1}$, respectively, in the spectrum of the substrate protected by BSB film (Fig. 3(iii)). The broad band at $3357 \mathrm{~cm}^{-1}$ are due to the $\mathrm{OH}$ - vibration from the adsorbed water molecules on the film surface which could not reach the substrate. The $\mathrm{N}-\mathrm{H}$ stretching bending vibrational band observed at $1591 \mathrm{~cm}^{-1}$ in the BSB spectrum was observed at $1634 \mathrm{~cm}^{-1}$ in that of the adsorbed BSB film. The reduced intensity of the $\mathrm{N}-\mathrm{H}, \mathrm{S}=\mathrm{O}$, and $\mathrm{C}-\mathrm{O}-\mathrm{C}$ vibrational bands in that spectrum, could be due to the interaction of the heteroatoms with the carbon steel surface. Hence, the BSB molecules could have been adsorbed onto the surface of the steel by that interaction. The aromatic ring can also be said to be involved in

Table 5 AFM parameters for the surfaces of bare polished carbon steel and carbon steel immersed room temperature $\left(25 \pm 1{ }^{\circ} \mathrm{C}\right)$ in the blank and BSB inhibited solutions

\begin{tabular}{llll}
\hline Sample & $\begin{array}{l}\text { Average roughness } \\
\left(R_{\mathrm{a}}\right)(\mu \mathrm{m})\end{array}$ & $\begin{array}{l}\text { RMS roughness } \\
\left(R_{\mathrm{q}}\right)(\mu \mathrm{m})\end{array}$ & $\begin{array}{l}\text { Maximum profile } \\
\text { valley depth }\left(R_{\mathrm{v}}\right)(\mu \mathrm{m})\end{array}$ \\
\hline CS immersed in blank 15\% HCl & 0.145 & 0.191 & 0.645 \\
CS immersed in 600 ppm BSB inhibited solution & 0.067 & 0.087 & 0.243
\end{tabular}




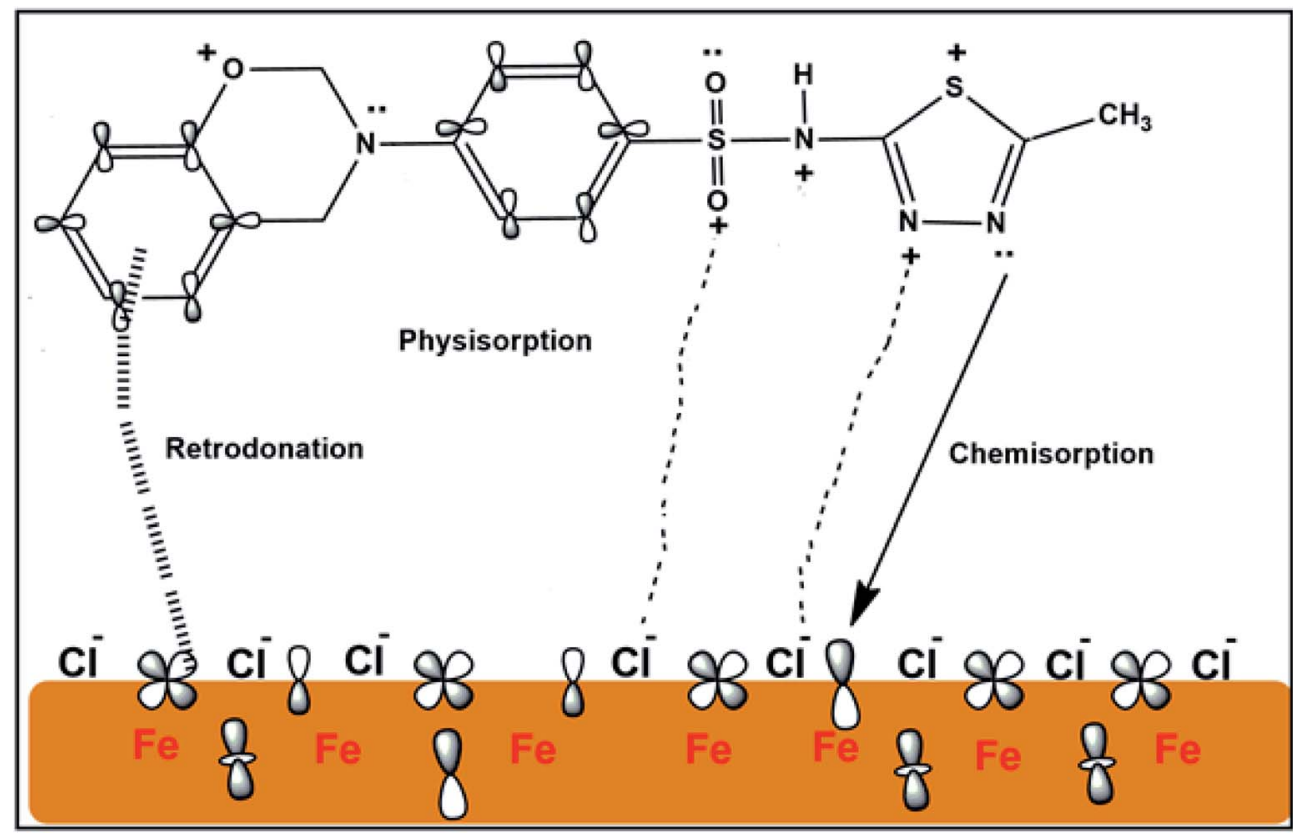

Fig. 14 Plausible mechanism of BSB adsorption on carbon steel surface.

another interaction with the steel surface as the intensity of the aromatic ring stretching vibrational band around $1500 \mathrm{~cm}^{-1}$ was also significantly lower in the adsorbed BSB film when compared to that of pure BSB.

\subsection{Mechanism of BSB inhibition}

Inhibitor molecules generally get adsorbed to metal surfaces either via physisorption, chemisorption, or both mechanisms. ${ }^{7,50}$ Inhibitor molecules can also interact with the steel surface via the retro-donation mechanism where excess delectrons from the steel surface, are donated to the inhibitor $\pi$ anti-bonding orbitals. ${ }^{51,52}$ From the PDP and the adsorption isotherm results, the BSB molecules could have been adsorbed on the surface of the steel via both the mechanisms of chemisorption and physisorption, with the physisorption being predominant. From the FTIR analysis, we could also suggest a retro-donation adsorption mechanism of the BSB molecules. A plausible mechanism for the adsorption of the BSB molecules onto the steel surface is schematically described in Fig. 14.

\section{Conclusion}

The mitigation of the corrosion of X60 carbon steel, in 15\% HCl that simulates an oil well acidizing environment by a novel synthesized benzenesulfonamide-based benzoxazine (BSB) inhibitor was evaluated. The evaluation was achieved, by the weight loss and electrochemical measurement techniques at room $(25 \pm 1)$, and higher $\left(40 \& 60^{\circ} \mathrm{C}\right)$ temperatures. The BSB inhibitor was characterized, by the FTIR, ${ }^{1} \mathrm{H}$ and ${ }^{13} \mathrm{C}$ NMR techniques. The SEM and AFM characterizations were used to examine the surface morphology of the carbon steel surface in the absence and presence of BSB inhibitor, while the FTIR and
EDS tools were used, to analyze the formed film on the carbon steel surface in the absence and presence of BSB. BSB efficiency increases from $88.36 \%$ at room temperature to $90.53 \%$ at $40{ }^{\circ} \mathrm{C}$ and $91.43 \%$ at $60{ }^{\circ} \mathrm{C}$ for its $600 \mathrm{ppm}$ concentration. A single active compound alone is usually not effective enough as a corrosion inhibitor, a blend of active compounds, together with surfactants, intensifiers, emulsifiers, and solvents to give 'corrosion inhibitor formulation' are normally used as inhibitors in an acidizing environment. For BSB, a single active compound exhibiting an efficiency of about $90 \%$ with a low corrosion rate (4.561 mm per year) at $60{ }^{\circ} \mathrm{C}$ far below the acceptable corrosion rate (50.08 $\mathrm{mm}$ per year), for the high temperature, acidizing solution, is an excellent performance. The PDP technique suggested the BSB acted as a mixed-type inhibitor with a predominant cathodic mechanism. The outcomes from the electrochemical analyses, surface morphological investigations (SEM/EDS, AFM), and the results of FT-IR analyses confirmed the corrosion inhibition performance of BSB over the steel surface. The adsorption follows the Langmuir adsorption theory with physisorption adsorption mechanism predominance.

\section{Conflicts of interest}

There is no conflicts of interest to declare.

\section{References}

1 L. Keshav, V. S. Teja and J. Vairamuthu, Performance analysis on synthesized reinforced carbon steel for structural applications, Mater. Today: Proc., 2020, 33, 44984500 . 
$2 \mathrm{~J}$. Tian, et al., Effect of annealing on the microstructure and mechanical properties of a low-carbon steel with ultrafine grains, Metallogr., Microstruct., Anal., 2017, 6(3), 233-239.

3 M. Migahed, et al., Corrosion inhibition behavior of synthesized imidazolium ionic liquids for carbon steel in deep oil wells formation water, Journal of Bio- and TriboCorrosion, 2017, 3(2), 22.

4 T. P. Rajendran, N. R. J. Hynes and T. Christopher, Characterization of high-carbon high-chromium tool steel/ low-carbon steel friction-welded joints for industrial tooling applications, J. Braz. Soc. Mech. Sci. Eng., 2018, 40(6), 316.

5 A. Yavuz, K. Kaplan and M. Bedir, Annealing of electrodeposited nickel on low carbon steel for supercapacitor applications, Dig. J. Nanomater. Bios., 2019, 14(4), 1061-1068.

6 K. R. Ansari, et al., Corrosion Inhibitors for Acidizing Process in Oil and Gas Sectors. Corrosion Inhibitors in the Oil and Gas Industry, 2020, pp. 151-176.

$7 \mathrm{~K}$. Haruna, et al., Gelatin: a green corrosion inhibitor for carbon steel in oil well acidizing environment, J. Mol. Liq., 2018, 264, 515-525.

8 P. Rajeev, A. Surendranathan and C. S. Murthy, Corrosion mitigation of the oil well steels using organic inhibitors a review, J. Mater. Environ. Sci., 2012, 3(5), 856-869.

$9 \mathrm{~K}$. Tamalmani and H. Husin, Review on Corrosion Inhibitors for Oil and Gas Corrosion Issues, Appl. Sci., 2020, 10(10), 3389.

10 O. Fayomi, I. Akande and S. Odigie, Economic Impact of Corrosion in Oil Sectors and Prevention: An Overview, in Journal of Physics: Conference Series, IOP Publishing, 2019.

11 R. G. M. de Araújo Macedo, et al., Water-soluble carboxymethylchitosan used as corrosion inhibitor for carbon steel in saline medium, Carbohydr. Polym., 2019, 205, 371-376.

12 U. Naresh, et al., Conducting Polymer Coatings for Corrosion Resistance in Electronic Materials, Polymers Coatings: Technology and Applications, 2020, p. 175.

$13 \mathrm{~W}$. Wei, et al., High temperature oxidation and corrosion behaviours of $\mathrm{Ni}-\mathrm{Fe}-\mathrm{Cr}$ alloys as inert anode for aluminum electrolysis, Corros. Sci., 2019, 157, 382-391.

14 A. O. Ijaola, P. K. Farayibi and E. Asmatulu, Superhydrophobic coatings for steel pipeline protection in oil and gas industries: a comprehensive review, J. Nat. Gas Sci. Eng., 2020, 83, 103544.

15 L. T. Popoola, et al., Corrosion problems during oil and gas production and its mitigation, Int. J. Ind. Chem., 2013, 4(1), 35.

16 B. D. B. Tiu and R. C. Advincula, Polymeric corrosion inhibitors for the oil and gas industry: design principles and mechanism, React. Funct. Polym., 2015, 95, 25-45.

17 H. Hayatdavoudi and M. Rahsepar, A mechanistic study of the enhanced cathodic protection performance of graphene-reinforced zinc rich nanocomposite coating for corrosion protection of carbon steel substrate, J. Alloys Compd., 2017, 727, 1148-1156.
18 L. Guo, et al., Toward understanding the adsorption mechanism of large size organic corrosion inhibitors on an $\mathrm{Fe}(110)$ surface using the DFTB method, RSC Adv., 2017, 7(46), 29042-29050.

19 T. I. Oguntade, et al., A Binary Mixture of Sesame And Castor Oil as an Ecofriendly Corrosion Inhibitor of Mild Steel In Crude Oil, Open Chem. Eng. J., 2020, 14(1), 25-35.

$20 \mathrm{M}$. El-Naggar, Corrosion inhibition of mild steel in acidic medium by some sulfa drugs compounds, Corros. Sci., 2007, 49(5), 2226-2236.

21 P. Mourya, et al., Relationship between structure and inhibition behaviour of quinolinium salts for mild steel corrosion: experimental and theoretical approach, Corros. Sci., 2015, 95, 71-87.

22 L. Guo, et al., Theoretical insight into an empirical rule about organic corrosion inhibitors containing nitrogen, oxygen, and sulfur atoms, Appl. Surf. Sci., 2017, 406, 301-306.

$23 \mathrm{H}$. Ishida, Overview and historical background of polybenzoxazine research, in Handbook of benzoxazine resins, Elsevier, pp. 3-81, 2011.

24 F. W. Holly and A. C. Cope, Condensation products of aldehydes and ketones with $o$-aminobenzyl alcohol and $o$ hydroxybenzylamine, J. Am. Chem. Soc., 1944, 66(11), 18751879.

25 V. Hemapriya, et al., Dry and wet lab analysis on benzofused heterocyclic compounds as effective corrosion inhibitors for mild steel in acidic medium, J. Ind. Eng. Chem., 2016, 40, 106-117.

26 G3-89A, Standard practice for conventions applicable to electrochemical measurements in corrosion testing, 2010, p. 1-9.

27 F. Shan, et al., Non-flammable thiazole-functional monobenzoxazines: synthesis, polymerization, thermal and thermomechanical properties, and flammability studies, Polymer, 2018, 157, 38-49.

28 N. E. A. Abdel-Sattar, A. M. El-Naggar and M. S. A. AbdelMottaleb, Novel Thiazole Derivatives of Medicinal Potential: Synthesis and Modeling, J. Chem., 2017, 2017, 4102796.

29 C. Fang, et al., Facile synthesis of alkaline-earth metal manganites for the efficient degradation of phenolic compounds via catalytic ozonation and evaluation of the reaction mechanism, J. Colloid Interface Sci., 2019, 551, 164-176.

30 S. Karakuş, et al., Synthesis, anticancer activity and ADMET studies of $N$-(5-methyl-1,3,4-thiadiazol-2-yl)-4-[(3substituted) ureido/thioureido] benzenesulfonamide derivatives, Phosphorus, Sulfur Silicon Relat. Elem., 2018, 193(8), 528-534.

$31 \mathrm{~F}$. Khallouki, et al., Isolation of the Major Phenolic Compounds in the Pits of Brined Green Olive Drupes: Structure Elucidation by Comprehensive 1H/13C-NMR Spectroscopy, Nat. Prod. Commun., 2019, 14(7), 1-8.

32 R. Andreu and J. Ronda, Synthesis of 3,4-dihydro- $2 \mathrm{H}-1,3-$ benzoxazines by condensation of 2-hydroxyaldehydes and primary amines: application to the synthesis of hydroxy- 
substituted and deuterium-labeled compounds, Synth. Commun., 2008, 38(14), 2316-2329.

$33 \mathrm{M}$. Ghandi and A. Olyaei, Evidences of some unusual behaviours of 2-aminothiazol and 2-aminobenzothiazol in reactions with formaldehyde and glyoxal, J. Heterocycl. Chem., 2007, 44(2), 323-327.

34 S.-A. Gârea, et al., Thermal polymerization of benzoxazine monomers followed by GPC, FTIR and DETA, Polym. Test., 2007, 26(2), 162-171.

35 N. Ghosh, B. Kiskan and Y. Yagci, Polybenzoxazines-new high performance thermosetting resins: synthesis and properties, Prog. Polym. Sci., 2007, 32(11), 1344-1391.

36 L. Han, et al., Oxazine ring-related vibrational modes of benzoxazine monomers using fully aromatically substituted, deuterated, $15 \mathrm{~N}$ isotope exchanged, and oxazine-ring-substituted compounds and theoretical calculations, J. Phys. Chem. A, 2017, 121(33), 6269-6282.

37 K. Haruna, T. A. Saleh and M. Quraishi, Expired metformin drug as green corrosion inhibitor for simulated oil/gas well acidizing environment, J. Mol. Liq., 2020, 315, 113716.

38 B. Chambers and D. Gambale, Material selection in strong hydrogen sulfide acidizing environments, Stainl. Steel World, 2011, 23, 29-35.

39 I. B. Onyeachu, I. B. Obot and A. Y. Adesina, Green corrosion inhibitor for oilfield application II: The time-evolution effect on the sweet corrosion of API X60 steel in synthetic brine and the inhibition performance of 2-(2-pyridyl) benzimidazole under turbulent hydrodynamics, Corros. Sci., 2020, 168, 108589.

40 A.-R. I. Mohammed, et al., Evaluation of the corrosion inhibition efficacy of Cola acuminata extract for low carbon steel in simulated acid pickling environment, Environ. Sci. Pollut. Res., 2020, 27(27), 34270-34288.

41 S. Allen, G. Mckay and J. F. Porter, Adsorption isotherm models for basic dye adsorption by peat in single and binary component systems, J. Colloid Interface Sci., 2004, 280(2), 322-333.

42 N. A. Odewunmi, et al., L-citrulline: an active corrosion inhibitor component of watermelon rind extract for mild steel in HCl medium, J. Taiwan Inst. Chem. Eng., 2015, 51, 177-185.

43 E. A. Noor and A. H. Al-Moubaraki, Thermodynamic study of metal corrosion and inhibitor adsorption processes in mild steel/1-methyl-4 [4'(-X)-styryl pyridinium iodides/ hydrochloric acid systems, Mater. Chem. Phys., 2008, 110(1), 145-154.

44 D. Dwivedi, K. Lepková and T. Becker, Carbon steel corrosion: a review of key surface properties and characterization methods, RSC Adv., 2017, 7(8), 4580-4610.

45 Z. Belarbi, et al., Study of Adsorption of Corrosion Inhibitor 1(2-aminoethyl)-2-oleyl-2-imidazolinium Chloride on Carbon Steel Under $\mathrm{CO}_{2}$ Environment by Using In Situ AFM Measurements, CORROSION/2017, 2017, p. 0990.

46 Y. Lei, et al., Adsorption and characterization of an organic corrosion inhibitor for inhibiting carbon steel corrosion in chloride solution, Curr. Anal. Chem., 2016, 12(2), 141-146.

47 V. Pandarinathan, et al., Adsorption of corrosion inhibitor 1dodecylpyridinium chloride on carbon steel studied by in situ AFM and electrochemical methods, Ind. Eng. Chem. Res., 2014, 53(14), 5858-5865.

48 K. Haruna, et al., Cyclodextrin-based functionalized graphene oxide as an effective corrosion inhibitor for carbon steel in acidic environment, Prog. Org. Coat., 2019, 128, 157-167.

49 M. Z. H. Khan, et al., The role of drug as corrosion inhibitor for mild steel surface characterization by SEM, AFM, and FTIR, Anti-Corros. Methods Mater., 2016, 63(4), 308-315.

50 I. Obot, et al., 8-Hydroxyquinoline as an alternative green and sustainable acidizing oilfield corrosion inhibitor, Sustainable Mater. Technol., 2017, 14, 1-10.

51 A. Espinoza-Vázquez, et al., Adsorption and corrosion inhibition behaviour of new theophylline-triazole-based derivatives for steel in acidic medium, R. Soc. Open Sci., 2019, 6(3), 181738.

52 T. Peme, et al., Adsorption and corrosion inhibition studies of some selected dyes as corrosion inhibitors for mild steel in acidic medium: gravimetric, electrochemical, quantum chemical studies and synergistic effect with iodide ions, Molecules, 2015, 20(9), 16004-16029. 